\title{
DEFORMATIONS OF GENERALIZED CALIBRATIONS AND COMPACT NON-KÄHLER MANIFOLDS WITH VANISHING FIRST CHERN CLASS *
}

\author{
JAN GUTOWSKI ${ }^{\dagger}$, STEFAN IVANOV $\ddagger$, AND GEORGE PAPADOPOULOS $§$
}

\begin{abstract}
We investigate the deformation theory of a class of generalized calibrations in Riemannian manifolds for which the tangent bundle has reduced structure group $U(n), S U(n), G_{2}$ and $\operatorname{Spin}(7)$. For this we use the property of the associated calibration form to be parallel with respect to a metric connection which may have non-vanishing torsion. In all these cases, we find that if there is a moduli space, then it is finite dimensional. We present various examples of generalized calibrations that include almost hermitian manifolds with structure group $U(n)$ or $S U(n)$, nearly parallel $G_{2}$ manifolds and group manifolds. We find that some Hopf fibrations are deformation families of generalized calibrations. In addition, we give sufficient conditions for a hermitian manifold $(M, g, J)$ to admit Chern and Bismut connections with holonomy contained in $S U(n)$. In particular we show that any connected sum of $k \geq 3$ copies of $S^{3} \times S^{3}$ admits a hermitian structure for which the restricted holonomy of a Bismut connection is contained in $S U(3)$.
\end{abstract}

1. Introduction. Riemannian manifolds with structure group a subgroup of an orthogonal group under mild topological assumptions admit a connection for which its reduced holonomy is a subgroup of the structure group. This connection is not necessarily the Levi-Civita connection but it may have non-vanishing torsion. The existence of such a connection with reduced holonomy a subgroup of an orthogonal group does not imply other geometric properties on a Riemannian manifold, like for example irreducibility. This is unlike the well-known case that involves the reduction of the holonomy group of the Levi-Civita connection which has led to Berger's classification list. Nevertheless the question arises as to whether the reduction of the structure group of a Riemannian manifold is related to some underlying geometric structure.

The aim of this paper is three-fold. First, we shall show that Riemannian manifolds which admit a metric connection with holonomy an appropriate subgroup of the orthogonal group may have submanifolds which are calibrated with respect to a generalized calibration. Second we shall investigate the moduli space of these calibrated submanifolds. Finally, we shall show the existence of a large class of hermitian manifolds with trivial canonical bundle which admit either a Chern or a Bismut connection which has reduced holonomy contained in $S U(n)$. Our latter result can be though off as a generalization of the Calabi-Yau theorem in the context of hermitian manifolds which are not Kähler.

Generalized calibrations were introduced by Gutowski and Papadopoulos [17] and further investigated in [18] to describe the solitons of brane actions with a nonvanishing Wess-Zumino term. These solitons are certain submanifolds which minimize an energy functional and are associated with calibration forms. These forms, unlike the case of standard calibrations, are not closed. In what follows we shall use the term generalized calibration to refer to both the calibration form and the calibrated submanifold. The distinction between the two will be clear from the context.

\footnotetext{
${ }^{*}$ Received December 12, 2002; accepted for publication December 27, 2002.

†Department of Physics, Queen Mary University of London, Mile End, London E1 4NS, UK.

$\ddagger$ Department of Mathematics, University of Sofia, "St. Kl. Ohridski"

${ }^{\S}$ Department of Mathematics, King's College London, Strand, London WC2R 2LS, UK (gpapas@mth.kcl.ac.uk).
} 
In this paper, we shall demonstrate that generalized calibrations arise in the investigation of manifolds that admit a metric connection with possibly non-vanishing torsion which has holonomy an appropriate subgroup of the orthogonal group. Although, generalized calibrations can be investigated independently, their use in manifold theory becomes more transparent in the context of holonomy groups and in manifolds with reduced structure group. This is because for certain holonomy groups, like for example those that occur in Berger's list; $U(n)(2 \mathrm{n}), S U(n)(2 \mathrm{n}), S p(n) \cdot S p(1)(4 \mathrm{n})$, $S p(n)(4 \mathrm{n}), G_{2}(7)$ and $\operatorname{Spin}(7)(8)$, manifolds admit parallel calibration forms which however are not necessarily closed; in parenthesis we have denoted the real dimension of the associated manifolds. Such forms give rise to generalized calibrated submanifolds which are minima of the energy functional

$$
E(Z)=\operatorname{Vol}(Z)-\int_{Z} \psi
$$

where $Z$ is a k-dimensional submanifold and $\psi$ is a calibration form of degree $k$, $d \psi \neq 0$. The submanifolds that minimize $E$ are not necessarily minimal.

We shall focus our investigation to the generalized calibrations associated with the holonomy groups $U(n)(2 \mathrm{n}), S U(n)(2 \mathrm{n}), G_{2}(7)$ and $\operatorname{Spin}(7)(8)$. We shall show that in most of these cases, the differential system associated with the deformation of the above generalized calibrations is elliptic. So if the moduli space of a generalized calibration exists, then it is finite dimensional. We shall not investigate the obstruction theory; this will appear in another publication. We shall also compute the second variation of the energy functional. The differential systems that arise in the deformation of generalized calibrations will also be investigated for various classes of manifolds that admit connections with the above holonomy. We shall see that in some cases they become simplified. We shall also give a large number of generalized calibrations as submanifolds of group manifolds, complex manifolds and homogeneous spaces. In particular, we shall show that some Hopf fibrations are families of generalized calibrations.

In the second part of the paper we shall focus on hermitian manifolds with vanishing first Chern Class. This is because they are a generalization of Calabi-Yau manifolds. Recently such manifolds have found applications in the investigation of Reid's conjecture and of mirror symmetry. This conjecture can be stated as follows: Let $X$ be a three-dimensional Calabi-Yau manifold and suppose that $X$ can be blown down along a rational curve to a possibly singular manifold $Y_{1}$. Such singularities of Calabi-Yau manifolds can be removed by a small deformation. Let $\tilde{Y}_{1}$ be the smooth deformation of $Y$. Now $Y_{1}$ has trivial canonical bundle and $b_{2}\left(\tilde{Y}_{1}\right)=b_{2}(X)-1$. Continuing this procedure, we shall end up with a smooth manifold $\tilde{X}$ with trivial canonical bundle and $b_{2}(\tilde{X})=0$. So $\tilde{X}$ cannot be Kähler. The conjecture is that if $X$ and $Z$ are Calabi-Yau manifolds with $b_{3}(X)=b_{3}(Z)$, then $\tilde{Z}$ is in the same deformation class of $\tilde{X}$.

The canonical bundle of a hermitian manifold can be topologically but not holomorphically trivial. For the definition of the former we take that the first Chern class vanishes. For the definition of the latter, we take that the canonical bundle admits a nowhere vanishing holomorphic section. We remark that there are canonical bundles which are topologically but not holomorphically trivial, such as the canonical bundle of $S U(3)$. It was discovered recently by Hitchin [20] that complex three-folds with holomorphically trivial canonical bundle appear as critical points of a certain diffeomorphism invariant functional on the space of differential three-forms on a closed six-dimensional manifold. 
There are several connections on hermitian manifolds compatible with both the hermitian metric and complex structure which coincide with the Levi-Civita connection in the Kähler case. Amongst these connections the Chern connection is the unique connection for which the torsion 2 -form is of type $(2,0)+(0,2)$, and the Bismut connection for which the torsion is a three form. If the torsion 3-form of the Bismut connection is closed or equivalently the Kähler form is $\partial \bar{\partial}$-closed, then the hermitian structure is said to be strong. The latter connection was used by Bismut [5] to prove a local index formula for the Dolbeault operator when the manifold is not Kähler but strong; vanishing theorems for the Dolbeault cohomology on a compact Hermitian non-Kähler manifold were found [3, 21, 22]. For other applications of the Bismut connection see [33, 21, 22]. In particular in [22] obstructions have been found to the Hodge numbers $h^{0,1}, h^{0, n}$ for hermitian manifolds whose Bismut connection has reduced holonomy contained in $S U(3)$.

Given a hermitian manifold $(M, g, J)$ with vanishing first Chern class, we give some sufficient conditions for $(M, g, J)$ to admit a Chern or a Bismut connection with restricted holonomy contained in $S U(n)$. The main tool that we shall use for the investigation of hermitian manifolds with trivial canonical bundle is the $\partial \bar{\partial}$-lemma. This lemma is valid for any compact Kähler manifold but there are non-Kähler spaces satisfying the $\partial \bar{\partial}$-lemma. A result of Deligne states that any Moishezon manifold is cohomologically Kähler and therefore it satisfies the $\partial \bar{\partial}$-lemma. The $\partial \bar{\partial}$-lemma also holds for any compact non-Kähler 3-fold with holomorphically trivial canonical bundle which is diffeomorphic to connected sums of $k \geq 2$-copies of $S^{3} \times S^{3}$ [28]. One of our main goals is to prove the following

THEOREM 1. On a connected sum of $k \geq 2$-copies of $S^{3} \times S^{3}$ there exists a hermitian structure for which the holonomy of the Bismut connection is contained in $S U(3)$. Such a hermitian structure is not strong.

The celebrated Yau's solution of the Calabi conjecture [35] states that on a 2ndimensional compact complex manifold with vanishing first Chern class of Kähler type there exists a Kähler metric with restricted holonomy contained in SU(n) (Ricci flat Kähler metric). For non-Kähler manifolds, it appears that the following holds:

CONJECTURE 1 . On any 2n-dimensional compact complex manifold $(n>2)$ with vanishing first Chern class there exists a hermitian structure with restricted holonomy of the Bismut connection contained in $S U(n)$.

The condition $n>2$ on the dimension in this statement is essential since the Inoue surface has vanishing first Chern class but it does not admit hermitian structure with $S U(2)$ holonomy of the Bismut connection as has been observed in [22].

Clearly this conjecture is true for connected sums of $k \geq 2$-copies of $S^{3} \times S^{3}$ in view of Theorem 1. It is also true for Moishezon manifolds and for compact complex manifolds with vanishing first Chern class which are cohomologically Kähler as we demonstrate in sections 17 and 18 below.

This paper has been organized as follows: In sections two and three, we give the definition of generalized calibrations and introduce the energy functional. In section four, we compute the second variation of the energy functional and demonstrate the relation between generalized calibrations and reduced holonomy. In section five, we examine the deformations of a class of almost hermitian calibrations. In section six, we derive the deformation equations of SAS calibrations and in section seven, we explore them in various special cases. In section eight, we give many examples of SAS calibrations and deformation families. In section nine, we give the deformation equations of generalized co-associative calibrations. In section ten, we give 
the deformation equations of generalized associative calibrations. In section eleven, we investigate the deformation equations of generalized associative and co-associative calibrations in various manifolds with special $G_{2}$ structures. In section twelve, we give many examples of associative and co-associative calibrations that include various deformation families. Some Hopf fibrations are such deformation families. In section thirteen, we give the deformation equations of generalized Cayley calibrations and in section fourteen we give a group manifold example. In section fifteen, we summarize some useful formulae for hermitian manifolds. In section sixteen, we investigate the existence of Chern connections with holonomy $S U(n)$ on hermitian manifolds with trivial canonical bundles and in section seventeen we investigate the existence of Bismut connections with holonomy $S U(n)$ on hermitian manifolds with trivial canonical bundles. In section eighteen, we give the proof of theorem one and in section nineteen, we give examples of manifolds with the holonomy of Bismut connection contained in $S U(3)$.

Acknowledgements. We would like to thank T.Pantev and V. Tsanov for helpful discussions. J.G. is supported by an EPSRC postdoctoral grant. S.I. is partially supported by Contract MM 809/1998 with the Ministry of Science and Education of Bulgaria, Contract 353/2000 with the University of Sofia "St. Kl. Ohridski". S.I. is a member of the EDGE, Research Training Network HPRN-CT-2000-00101, supported by the European Human Potential Programme. G.P. is supported by Royal Society University Research fellowship. Part of this work was done while one of us G.P. was participating at the M-theory programme of the Newton Institute.

2. Generalized calibrations and relative de Rham Cohomology. Here we shall describe some of the main properties of generalized calibrations using relative de Rham cohomology. Generalized calibrations were defined in [17] in the context of understanding the solitons of brane actions with a Wess-Zumino term. In manifold theory, these solitons are certain submanifolds of a manifold which admits an appropriate form. These submanifolds are not minimal but they are the minima of a certain energy functional. We begin with a definition of generalized calibrations and in particular of the energy functional.

DEFINITION. A generalized calibration of degree $k$ is a $\mathrm{k}$-form $\phi$ on an oriented manifold $M$ which satisfies at every point $p$ the inequality $\left.\phi(\xi)\right|_{p} \leq 1$ for every oriented k-plane $\xi$ in $T_{p} M$.

For standard calibrations it is assumed in addition that $\phi$ is closed, $d \phi=0$. This is not the case here.

Definition. The contact set $C_{p}(\phi)$ at a point $p \in M$ of a calibration $\phi$ is

$$
C_{p}=\left\{\xi \in G r\left(k, T_{p} M\right): \phi(\xi)=1\right\} .
$$

For calibrations of interest the contact sets are not empty.

Definition. Generalized calibrated submanifolds $X$ of $M$ are those for which $\phi\left(T_{p} X\right)=1$ at every $p \in X$.

In what follows we shall refer to both $\phi$ and $X$ as "generalized calibrations" of $M$. The distinction between the two will be clear from the context.

Generalized calibrations minimize a family of functionals [17]. Here we shall repeat the analysis using relative homology and relative de Rham cohomology. Let 
$M$ be a manifold and $N$ a submanifold of $M$ with $\operatorname{dim} N \geq k$. Suppose that $(\alpha, \beta)$ is a pair of forms such that $\alpha \in \Omega^{k+1}(M)$ and $\beta \in \Omega^{k}(N)$. In addition assume that $\beta$ is a calibration. Next suppose that $K$ is a submanifold of $N$. Take an open ball $D \subset K$ and consider the functional

$$
\mathcal{E}(D, L)=\operatorname{Vol}(D)-\int_{L} \alpha
$$

where $L$ is a submanifold of $M$ such that $\partial L=D+Y$. We shall refer to $\mathcal{E}$ as the energy of $D$.

We shall show that the functional $\mathcal{E}$ is minimized whenever $D \subset X$ and $X$ is a calibrated submanifold of $N$. However before we proceed to show this consider $D_{1}$ and $D_{2}$ two open balls in $N$ such that $\partial D_{1}=\partial D_{2}$. Then we have

$$
\int_{D_{1}} \beta-\int_{L_{1}} \alpha-\left(\int_{D_{2}} \beta-\int_{L_{2}} \alpha\right)=\int_{S} \beta-\int_{Z} \alpha
$$

where $\partial L_{2}=Y+D_{2}$ and $S=D_{1}-D_{2}$ is the sphere in $N$ which can be constructed by gluing the discs $D_{1}$ and $D_{2}$ along the common boundary taking into account their relative orientations. In addition $Z$ is obtained by gluing $L_{1}$ and $L_{2}$ along $Y$ and so it has boundary $\partial Z=\partial L_{1}-\partial L_{2}=\left(Y+D_{1}\right)-\left(Y+D_{2}\right)=D_{1}-D_{2}=S$. So $Z$ is a cycle in $M$ relative to the submanifold $N$ and $Z \in H_{k+1}(M, N)$.

Now suppose that $(\alpha, \beta)$ represents a trivial class in the relative de Rham cohomology $H_{d R}^{k+1}(M, N)$. Recall that the cohomology operator $d$ in relative de Rham cohomology is defined as $d(\alpha, \beta)=\left(d \alpha,\left.\alpha\right|_{N}-d \beta\right)$. Therefore $[(\alpha, \beta)]$ is a trivial class iff $\alpha$ is exact, $\alpha=d \gamma$, which implies that $d\left(\left.\gamma\right|_{N}-\beta\right)=0$, and $\left.\gamma\right|_{N}-\beta$ is an exact form in $N,\left.\gamma\right|_{N}-\beta=d \zeta$. In such a case, we have

$$
\int_{S} \beta-\int_{Z} \alpha=0
$$

and so

$$
\int_{D_{1}} \beta-\int_{L_{1}} \alpha-\left(\int_{D_{2}} \beta-\int_{L_{2}} \alpha\right)=0 .
$$

TheOREM 2. Let $(\alpha, \beta)$ represent the trivial class in $H_{d R}^{k+1}(M, N)$ and $\beta$ be a calibration form in $N$. Then calibrated submanifolds $X$ of $N$ minimize the functional $\mathcal{E}\left(D_{1}, L_{1}\right)$. have

Proof. Let $X$ be a calibrated submanifold of $N$ and $D_{1}$ a disc in $X$. Then we

$$
\begin{aligned}
\mathcal{E}\left(D_{1}, L_{1}\right) & =\operatorname{Vol}\left(D_{1}\right)-\int_{L_{1}} \alpha \\
& =\int_{D_{1}} \beta-\int_{L_{1}} \alpha \\
& =\int_{D_{2}} \beta-\int_{L_{2}} \alpha \leq \operatorname{Vol}\left(D_{2}\right)-\int_{L_{2}} \alpha \\
& =\mathcal{E}\left(D_{2}, L_{2}\right) .
\end{aligned}
$$

The first equality follows from the definition of the functional. The second equality follows from the assumption that $X$ is calibrated. The third equality follows because 
the class $(\alpha, \beta)$ is trivial in $H_{d R}^{k+1}(M, N)$. Finally the inequality follows from the definition of generalized calibration.

Next we shall investigate some of the properties of the functional (2.3). In particular we have the following:

THEOREM 3. Let $K$ be a closed submanifold of $N \subset M$ and $L$ a submanifold of $M$ such that $\partial L=K$. In addition assume that $(\alpha, \beta)$ represent the trivial class in $H_{d R}(M, N)$. Then the functional $\mathcal{E}(K, L)$ is independent of the choice of $L$.

Proof. Let $L^{\prime}$ another submanifold of $M$ such that $\partial L^{\prime}=K$. Then we have

$$
\mathcal{E}\left(K, L^{\prime}\right)=\operatorname{Vol}(K)-\int_{L^{\prime}} \alpha=\operatorname{Vol}(K)-\int_{K} \gamma=\operatorname{Vol}(K)-\int_{L} \alpha=\mathcal{E}(K, L) .
$$

The first equality follows from the definition. The second equality follows from Stoke's theorem because $\alpha$ is exact and so $\alpha=d \gamma$. The third equality also follows for the same reason as the second, and the last follows from the definition of the functional $\mathcal{E}$.

REMARK 1. Suppose that $(\alpha, \beta)$ represents a class in $H^{k+1}(M, N ; \mathbb{Z})$. Then it is straightforward to see that the functional $\mathcal{E}(K, L) \bmod \mathbb{Z}$ is independent of the choice of $L$.

3. Special Cases. A special case of interest is whenever the generalized calibration form $\beta$ is defined as a generalized calibration on $M$. For $(\alpha, \beta)$ to be a trivial class, $\alpha=d \gamma, \alpha=d \beta$ and $\gamma-\beta=d \zeta$ must be an exact form in $M$. In such a case the functional $\mathcal{E}$ can be written as

$$
\mathcal{E}(D)=\operatorname{Vol}(D)-\int_{D} \gamma
$$

If $X$ is a compact calibrated submanifold of $M$ with boundary $\partial X$, then

$$
\mathcal{E}(X)=-\int_{\partial X} \zeta .
$$

In particular if $X$ is closed, then $\mathcal{E}(X)=0$. Since $\mathcal{E}(Z)>0$ if $Z$ is a closed but not calibrated submanifold of $M$, calibrated closed submanifolds of $M$ are global minima of $\mathcal{E}$. Another special case to consider is whenever $\alpha=d \gamma$, and we choose $\gamma=\beta$. This is the case which we shall focus on later. The functional $\mathcal{E}$ in this case is

$$
E(D)=\operatorname{Vol}(D)-\int_{D} \beta
$$

It is worth adapting the main theorem of generalized calibrations to this case. In particular we have the following:

THEOREM 4. Let $\beta$ be a generalized calibration in $M$; then calibrated submanifolds $X$ of $M$ minimize the functional $E$ in (3.11).

Proof. Let $X$ be a calibrated submanifold of $M$ and $D$ an open ball in $X$. Next let $D^{\prime}$ an open ball in $M$, such that $\partial D=\partial D^{\prime}$. Then we have

$$
E(D)=\operatorname{Vol}(D)-\int_{D} \beta=\int_{D} \beta-\int_{D} \beta=0=\int_{D^{\prime}} \beta-\int_{D^{\prime}} \beta \leq \operatorname{Vol}\left(D^{\prime}\right)-\int_{D^{\prime}} \beta=E\left(D^{\prime}\right) .
$$


The equalities are obvious. The inequality follows from the defining property of the calibration form.

The energy (3.11) vanishes when evaluated at every calibrated submanifold $X$ of $M$. In addition $E(Z)>0$ if $Z$ is not calibrated and therefore calibrated submanifolds are global minima of $E$. From now on, we shall focus on the calibrated submanifolds which are the minima of the functional (3.11).

REMARK 2. Standard calibrations for which the calibration form is closed $d \beta=0$ are special cases of the generalized calibrations associated with the functional (3.11). The only difference is that the energy functional used for standard calibrations is the induced volume Vol. Calibrated submanifolds under the standard calibrations are minimal. For the generalized calibrations the functional (3.11) is not the induced volume but nevertheless it can be identified with the "energy" of the submanifold. Observe that the relation between (3.11) and induced volume evaluated on a closed submanifold $Z$ of $M$ is

$$
E(Z)=\operatorname{Vol}(Z)-\beta[Z] .
$$

The last term depends only on the cohomology class of $\beta$. In particular it does not contribute in the equations for the criticality of $E$ and so the generalized calibrated submanifolds $X$ are minimal. Conversely, if $\beta$ is a closed form, then minimal submanifolds of $M$ minimize the energy functional $E$.

\section{Deformation of generalized calibrations.}

4.1. The second variation of the energy functional. Here we compute the second variation of the energy functional evaluated on a calibrated submanifold. Suppose $X$ is a calibrated submanifold of $M$ with respect to a generalized calibration $\phi$, and let $X(t)$ be a 1-parameter family of submanifolds of $M$ with $X(0)=X$. In addition let $d \operatorname{vol}(t) \equiv d \operatorname{vol}(X(t))$ be the volume form of $X(t)$, and $\phi(t)$ be the restriction of the calibration form on $X(t)$. Since $d \operatorname{vol}(t)$ and $\phi(t)$ are top forms on $X(t)$, we have $\phi(t)=\lambda(t) d \operatorname{vol}(t)$ for some function $\lambda(t)$ where $\lambda(0)=1$. This condition follows because the volume form of a calibrated submanifold is equal to the calibration form. Setting $E(t)=E(X(t))$, we have that the energy functional (3.11) is

$$
\begin{aligned}
E(0) & =\int_{X(0)} \phi-\int_{X(0)} \phi \\
& =\int_{X(t)} \phi-\int_{X(t)} \phi \\
& =\int_{X} \phi(t)-\int_{X} \phi(t) \\
& =\int_{X}(\lambda(t) d \operatorname{vol}(t)-\phi(t)) .
\end{aligned}
$$

Thus $\int_{X} \lambda(t) d \operatorname{vol}(t)-\phi(t)$ is independent of $t$. Differentiating the energy functional twice and evaluating at $t=0$, we obtain

$$
\left.\frac{d^{2}}{d t^{2}} E(t)\right|_{t=0}=-\left.\int_{X} \frac{d^{2}}{d t^{2}} \lambda(t)\right|_{t=0} d \operatorname{vol}(0)-\left.\left.2 \int_{X} \frac{d}{d t} \lambda(t)\right|_{t=0} \frac{d}{d t} d \operatorname{vol}(t)\right|_{t=0}
$$

where we have used $\lambda(0)=1$ and (4.14). To proceed with the computation of the second variation of the energy functional, we shall show that $\left.\frac{d}{d t} \lambda(t)\right|_{t=0}=0$. This a 
consequence of the calibration bound. In particular we have the following:

$$
d \operatorname{vol}(t)-\phi(t)=(1-\lambda(t)) d \operatorname{vol}(t) .
$$

Evaluating this on an appropriately oriented orthonormal basis and using the calibration bound, we find that $\lambda(t) \leq 1$. Since $\lambda(0)=1$ is a maximum, we have $\left.\frac{d}{d t} \lambda(t)\right|_{t=0}=0$.

To conclude, the second variation of the energy is

$$
\left.\frac{d^{2}}{d t^{2}} E(t)\right|_{t=0}=-\left.\int_{X} \frac{d^{2}}{d t^{2}} \lambda(t)\right|_{t=0} d \operatorname{vol}(0) .
$$

As in the case of standard calibrations, the second variation of the energy can be computed in terms of the normal vector field $V$. The proof is similar to that given in [29]. The result is summarized in the following theorem:

THEOREM 5 .

$$
\left.\frac{d^{2}}{d t^{2}} E(t)\right|_{t=0}=\int_{X}\left(\left\|\nabla^{\perp} V\right\|^{2} d \operatorname{vol}(0)-\nabla_{V}^{g} \nabla_{V}^{g} \phi-i_{\nabla^{\perp} V} i_{\nabla^{\perp} V} \phi-2 i_{\nabla^{\perp} V} \nabla_{V}^{g} \phi\right)
$$

where $\nabla_{V}^{g}$ is the Levi-Civita covariant derivative of $M$ along the normal direction $V$ of $X$ and $\nabla^{\perp} V$ is the covariant derivative of the normal bundle of $X$ in $M$ induced by the Levi-Civita connection of $M$.

4.2. Special holonomy and generalized calibrations. Let $(M, g)$ be a Riemannian manifold which admits a metric connection $\nabla$ with possibly non-vanishing torsion and holonomy contained in one of the groups $U(n)(2 n), S U(n)(2 n), S p(n)$ $(4 n), S p(1) \cdot S p(n)(4 n), G_{2}(7)$ and $\operatorname{Spin}(7)(8)$; the entry in $(\cdot)$ is the real dimension of $M$. Manifolds with such holonomy admit generalized calibration forms. These are forms parallel with respect to the connection $\nabla$. In what follows we shall not investigate all cases. Rather we shall focus on Riemannian manifolds $(M, g)$ which admit a metric connection $\nabla$ with possibly non-vanishing torsion and holonomy $U(n)(2 n)$, $S U(n)(2 n), G_{2}(7)$ and $\operatorname{Spin}(7)(8)$. The general theory of deformations of generalized calibrations will be developed without further assumptions. However in many examples that we shall present later, we shall require that $(M, g)$ satisfies some additional geometric conditions in addition to those that are a consequence of the reduction of the structure group of $T M$. These will simplify some aspects of the deformation theory of generalized calibrations and in particular the deformation equations. In particular we shall consider the following cases:

Holonomy $U(n)$

Suppose that a Riemannian manifold $(M, g)(\operatorname{dim} M=2 n)$ is equipped with a metric connection $\nabla$ whose holonomy is contained in $U(n)$. Then $M$ admits an almost complex structure $J, J^{2}=-1$, which is parallel with respect to $\nabla$ and the metric $g$ is hermitian with respect to $J, g(J X, J Y)=g(X, Y)$ for $X, Y$ vector fields on $M$. Therefore $(M, g, J)$ is an almost hermitian manifold with compatible connection $\nabla$. Conversely, let $(M, J, g)$ be an almost hermitian manifold, then $(M, J, g)$ admits a connection $\nabla$ with holonomy contained in $U(n)$. Such a connection $\nabla$ can be constructed from the Levi-Civita connection $\nabla^{g}$ of $g$ as

$$
\nabla_{X} Y=\nabla_{X}^{g} Y-\frac{1}{2} J\left(\nabla_{X}^{g} J\right) Y
$$


where $X, Y$ are vector fields on $M$. It is straightforward to verify that $g$ and $J$ are $\nabla$-parallel, $\nabla g=\nabla J=0$. Note that any almost hermitian manifold $(M, g, J)$ has a Kähler form $\Omega(X, Y)=g(X, J Y) . \Omega$ is $\nabla$-parallel but it is not closed, $d \Omega \neq 0$.

Proposition 1. Let $(M, g, J)$ be an almost hermitian manifold and $\Omega$ be the associated Kähler form. The forms $\phi_{k}=\frac{1}{k !} \Omega^{k}$ are generalized calibrations of degree 2k. The contact set at every point of $M$ is $G r\left(k, \mathbb{C}^{n}\right)$.

Proof. To show this, we shall demonstrate the above statement at a neighbourhood $U$ of a point $p \in M$. Then because $\phi_{k}$ is parallel, it will hold everywhere in $M$. We remark that there is a neighbourhood of a point $p \in M$ and a local frame $\left\{e^{a}, \epsilon^{\bar{a}} ; a=1, \ldots, n\right\},\left(e^{\bar{a}}=\left(e^{\bar{a}}\right)\right)$, of $(M, g, J)$ such that the metric and Kähler form can be written as

$$
\begin{aligned}
g & =\sum_{a, \bar{b}=1}^{n} \delta_{a \bar{b}} e^{a} e^{\bar{b}} \\
\Omega & =-i \sum_{a, \bar{b}=1}^{n} \delta_{a \bar{b}} e^{a} \wedge e^{\bar{b}} .
\end{aligned}
$$

Observe that in such a frame the metric and Kähler form take the standard form of a Euclidean metric and (almost) complex structure on $\mathbb{R}^{2 n}=\mathbb{C}^{n}$. It follows that $\phi_{k}$ are calibrations from Wirtinger's inequality on $\mathbb{R}^{2 n}$. From the same inequality it follows that the contact set consists of the complex k-planes in $\mathbb{R}^{2 n}=\mathbb{C}^{n}$. All the planes of the contact set can be constructed by acting with $U(n)$ on the k-plane

$$
\xi_{0}=\left\{\left(z_{1}, \ldots, z_{k}, 0, \ldots, 0\right): z_{1}, \ldots, z_{k} \in \mathbb{C}\right\} \subset \mathbb{C}^{n} .
$$

The calibrated submanifolds $X$ are almost hermitian submanifolds of $(M, g, J)$. Both the metric and almost complex structure on $X$ are induced from those on $M$; the almost complex structure on $X$ is induced from that on $M$ because at every point $p \in X$, the holomorphic subspace of $T_{p} X \otimes \mathbb{C}$ is identified with a complex k-plane of the contact set at $p$. Observe that the dimension of the contact set at every point is $2 k(n-1)$. We shall refer to these generalized calibrations as almost hermitian calibrations. These results can be summarized as follows:

COROLlaRY 1. The almost hermitian calibrations of degree $2 k$ of an almost hermitian manifold $(M, g, J)$ are almost hermitian submanifolds of real dimension $2 k$.

Suppose that $(M, g, J)$ is a hermitian manifold, i.e. the almost complex structure $J$ is integrable. It is known that such manifolds admit various connections $\nabla$ with non-vanishing torsion such that $\nabla g=\nabla J=0$. Because of this the holonomy of all such connections $\nabla$ is contained in $U(n)$. Again the forms $\phi_{k}=\frac{1}{k !} \Omega^{k}$ are generalized calibrations. The contact set at every point of $M$ is $\operatorname{Gr}\left(k, \mathbb{C}^{n}\right)$. The proof is identical to the one given above for the almost hermitian manifolds. In this case, the calibrated submanifolds $X$ are hermitian submanifolds of $(M, g, J)$. In particular, they are complex submanifolds. To show this observe that the Nijenhuis tensor of $X$ vanishes because the complex structure $J$ of $M$ is integrable. Such calibrations have been called hermitian calibrations in [17]. These results can be summarized as follows:

COROLlaRY 2. The hermitian calibrations of degree $2 k$ of a hermitian manifold $(M, g, J)$ are hermitian submanifolds of real dimension $2 k$. 
$\underline{\text { Holonomy } S U(n)}$

Suppose that a Riemannian manifold $(M, g)(\operatorname{dim} M=2 n)$ admits a metric connection $\nabla$ whose holonomy is contained in $S U(n)$. In such a case $(M, g)$ admits an almost complex structure $J$ such that $(M, g, J)$ is an almost hermitian manifold equipped with a $(\mathrm{n}, 0)$-form $\psi$ such that $\nabla g=\nabla J=\nabla \psi=0$. Compatibility of these conditions requires that the form $\psi$ is appropriately normalized. In particular

$$
(-1)^{\frac{1}{2}(n-1) n}\left(\frac{i}{2}\right)^{n} \psi \wedge \bar{\psi}=d \mathrm{vol}
$$

where $\bar{\psi}$ is the associated $(0, n)$ form and $d$ vol is the volume form of $M$ with respect to the metric $g$.

Conversely, let $(M, g, J)$ be an almost hermitian manifold with topologically trivial canonical bundle, then there is a connection $\nabla$ which has holonomy contained in $S U(n)$. To see this, observe that $(M, g, J)$ admits a no-where vanishing (n,0)-form $\psi$ but in general $\psi \wedge \bar{\psi}$ will not be related to the volume form of $M$ as in (4.22). In general, we have

$$
\psi \wedge \bar{\psi}=(-1)^{-\frac{1}{2}(n-1) n+n}(2 i)^{n} f^{2} d \operatorname{vol}_{\mathrm{g}}
$$

for some nowhere vanishing real-valued function $f$ of $M$, where $\bar{\psi}$ is the associated $(0, n)$ form. Now there are two possibilities to consider. First, define $\chi=f^{-1} \psi$. Observe that $\chi$ is again a nowhere vanishing section of the canonical bundle and it is normalized as in (4.22). In such case, one can show that the connection

$$
\nabla_{i} Y^{j}=\nabla_{i}^{g, J} Y^{j}+\frac{1}{n !} \chi^{j k_{1} \ldots k_{n-1}} \nabla_{i}^{g, J} \chi_{k k_{1} \ldots k_{n-1}} Y^{k}+\frac{1}{n !} \bar{\chi}^{j k_{1} \ldots k_{n-1}} \nabla_{i}^{g, J} \bar{\chi}_{k k_{1} \ldots k_{n-1}} Y^{k}
$$

has holonomy contained in $S U(n)$, i.e. $g, J$ and $\chi$ are all $\nabla$-parallel, where $\nabla^{g, J}$ is a connection with holonomy contained in $U(n), \nabla^{g, J} g=\nabla^{g, J} J=0$, such as the one given in (4.19).

Alternatively, observe that $f$ is either a positive or negative function. So without loss of generality we can take $f$ to be positive because if it is negative we can take $|f|$. Next define a new metric $h$ on $M$ by $h=f^{\frac{2}{n}} g$. Then observe that $(M, h, J)$ is again a hermitian manifold and the (n,0)-form $\psi$ is normalized as in (4.22) with respect to the new metric $h$. In such a case, one can show that the connection

$$
\nabla_{i} Y^{j}=\nabla_{i}^{h, J} Y^{j}+\frac{1}{n !} \psi^{j k_{1} \ldots k_{n-1}} \nabla_{i}^{h, J} \psi_{k k_{1} \ldots k_{n-1}} Y^{k}+\frac{1}{n !} \bar{\psi}^{j k_{1} \ldots k_{n-1}} \nabla_{i}^{h, J} \bar{\psi}_{k k_{1} \ldots k_{n-1}} Y^{k}
$$

has holonomy contained in $S U(n)$, ie $h, J$ and $\psi$ are all $\nabla$-parallel, where $\nabla^{h, J}$ is a connection with holonomy contained in $U(n), \nabla^{h, J} h=\nabla^{h, J} J=0$, such as the one given in (4.19) but constructed using the Levi-Civita connection of the metric $h$.

Proposition 2. Let $(M, g, J)$ be an almost hermitian manifold, $\operatorname{dim} M=2 n$, with trivial canonical bundle and associated parallel $(n, 0)$-form $\psi$. The form $\phi=$ $\operatorname{Re}(\psi)$ is a generalized calibration of degree $n$. The contact set $C_{p}$ at every point of $(M, g, J)$ is $S U(n) / S O(n)$.

Proof. As in the case of almost hermitian calibrations, we shall prove the proposition in a neighbourhood $U$ of a point $p \in M$. In such a neighbourhood, there is a 
local frame $\left\{e^{a}, e^{a^{\prime}}\right\}$ in $(M, g, J)$ such that

$$
\begin{aligned}
g & =\sum_{a=1}^{n}\left(\left(e^{a}\right)^{2}+\left(e^{a^{\prime}}\right)^{2}\right) \\
\Omega & =\sum_{a=1}^{n} e^{a} \wedge e^{a^{\prime}} \\
\psi & =\left(e^{1}+i e^{1^{\prime}}\right) \wedge \cdots \wedge\left(e^{n}+i e^{n^{\prime}}\right) .
\end{aligned}
$$

Again in this frame the metric, Kähler form and (n,0)-form take the standard form of those in $\mathbb{R}^{2 n}$. These are precisely the data of a SLAG calibration in $\mathbb{R}^{2 n}$. Therefore it follows that $\phi$ is a generalized calibration from the results of Harvey and Lawson as they apply for Special Lagrangian (SLAG) calibrations. Similarly, it follows that the contact set at every point $p \in M$ is $S U(n) / S O(n)$. All the calibrated planes can be constructed by acting with $S U(n)$ on the standard plane

$$
\xi_{0}=\left\{\left(x_{1}, \ldots, x_{n}, 0, \ldots, 0\right): x_{1}, \ldots, x_{n} \in \mathbb{R}\right\} \subset \mathbb{R}^{2 n} .
$$

Such generalized calibrations have been called Special Almost Symplectic or SAS for short [17]. The SAS calibrations are real middle dimension submanifolds of $(M, g, J)$. We remark that if $\operatorname{Re} \psi$ is a SAS calibration, then $\operatorname{Re}\left(e^{i \theta} \psi\right)$ is also a SAS calibration, where $\theta$ is a constant angle.

So if $(M, g, J)$ is an almost hermitian manifold with a compatible connection which has holonomy contained in $S U(n)$, then $M$ admits two types of generalized calibrations with calibrated submanifolds; the almost hermitian and the SAS. This is reminiscent of Calabi-Yau manifolds which have two types of calibrated submanifolds the Kähler and SLAG.

A special case that we shall investigate later is that in which $(M, g, J)$ is a hermitian manifold which admits a compatible connection $\nabla$ with holonomy contained in $S U(n)$. Again these manifolds admit two types of calibrations; the hermitian and the SAS. The contact set of SAS calibrations is $S U(n) / S O(n)$ at every point of $M$.

The above results are summarized as follows:

Corollary 3. Let $(M, g, J)$ be an (almost) hermitian manifold with trivial canonical bundle. Then $(M, g, J)$ admits (almost) hermitian and SAS calibrations.

Another class of hermitian manifolds $(M, g, J)$ are those for which the canonical bundle is holomorphically trivial. Connections on such manifolds will be investigated in the second part of this paper. Such manifolds admit a holomorphic $(n, 0)$-form $\psi$. Thus $\psi$ is closed, $d \psi=0$, but $\psi$ is not always normalized as in (4.22). In such a case, as we have explained, we can either rescale the form $\psi$ or we can rescale the metric $g$. In particular we can rescale the metric $g$ as $h=f^{\frac{2}{n}} g$ so that $\psi$ becomes a calibration form, where $f$ is given in (4.23). Since $\psi$ remains closed, the associated calibrated submanifolds are SLAGs and therefore minimal with respect to $h$. Alternatively as we have seen, one can rescale the holomorphic $(n, 0)$-form $\psi$ as $\chi=f^{-1} \psi$ where $f$ is given in (4.23). In this case, the rescaled form $\chi$ is a calibration but it is not closed. The associated calibrated submanifolds are SAS with respect to the original metric $g$.

Another special case that has recently been investigated is that of Kähler manifolds $(M, g, J)$ with trivial canonical bundle for which the metric $g$ is not a Calabi-Yau metric. Such manifolds have been called almost Calabi-Yau and have been studied in the context of mirror symmetry (see [24]). Note that as a consequence of the 
Calabi-Yau theorem, compact almost Calabi-Yau manifolds always admit a CalabiYau metric. Compact almost Calabi-Yau manifolds admit a holomorphic $(n, 0)$ form $\psi$, which is therefore closed $(d \psi=0)$, but not necessarily parallel with respect to a hermitian connection because it does not satisfy the normalization condition (4.22). The strategy adopted in this case is to conformally rescale the metric $g$, as in the case of hermitian manifolds with holomorphically trivial canonical bundle above, so that $\psi$ remains closed, and the associated calibrated submanifolds are Special Lagrangian and therefore minimal with respect to the rescaled metric. Alternatively as we have seen, one can rescale the holomorphic $(n, 0)$-form $\psi$. The associated calibrated submanifolds are SAS with respect to the original metric $g$.

In the case that $(M, g, J)$ is Calabi-Yau, then the hermitian calibrations become the standard Kähler calibrations while the SAS calibrations become the standard SLAG calibrations.

\section{$\underline{\text { Holonomy } G_{2}}$}

A Riemannian manifold $(M, g)(\operatorname{dim} M=7)$ equipped with a metric connection $\nabla$ whose holonomy is contained in $G_{2}$ admits a $\nabla$-parallel three-form $\psi$ and a $\nabla$ parallel four-form $* \psi$ which is the dual of $\psi$. As we shall show these forms $\psi$ and $* \psi$ are generalized associative and generalized co-associative calibrations, respectively.

Conversely, let $(M, g, \psi)$ be a Riemannian manifold which admits a three-form $\psi$ that satisfies the algebraic conditions of a $G_{2}$ invariant structure (such a three-form is stable in the terminology of [20]), then there is a connection $\nabla$ which has holonomy $G_{2}$. This connection can be expressed in terms of the Levi-Civita connection $\nabla^{g}$ of $g$ and the form $\psi$ as

$$
\nabla_{k} Y^{i}=\nabla_{k}^{g} Y^{i}+\frac{1}{18} \psi_{p q}^{i} \nabla_{k}^{g} \psi_{j}^{p q} Y^{j}+\frac{1}{108} * \psi_{p q r}^{i} \nabla_{k}^{g} * \psi_{j}^{p q r} Y^{j}
$$

where $Y$ is a vector field.

Proposition 3. Let $(M, g, \psi)$ a seven-dimensional manifold which admits a $G_{2}$ structure as above. The forms $\psi$ and $* \psi$ are generalized calibrations of degree three and four, respectively. In both cases the contact set at every point of $M$ is $G_{2} / S O(4)$.

Proof. To show this, we remark that there is locally a frame $\left\{e^{A} ; A=1, \ldots, 7\right\}$ of $(M, g, \psi)$ such that the metric and the $\nabla$-parallel three-form $\psi$ can be written as

$$
\begin{aligned}
& g=\sum_{A=1}^{7}\left(e^{A}\right)^{2} \\
& \psi=e^{123}+e^{1} \wedge\left(e^{45}-e^{67}\right)+e^{2} \wedge\left(e^{46}+e^{57}\right)+e^{3} \wedge\left(e^{47}-e^{56}\right),
\end{aligned}
$$

where $e^{12}=e^{1} \wedge e^{2}$ and similarly for the rest. Observe that in such a frame the metric $g$ and parallel three-form $\psi$ take the standard form of a Euclidean metric and $G_{2^{-}}$ invariant three-form in $\mathbb{R}^{7}$. It follows that both $\psi$ and $* \psi$ are generalized calibrations from the results of Harvey and Lawson as they apply for associative and co-associative calibrations. It also follows that the contact set at every point of $M$ is $G_{2} / S O(4)$. All the planes of the contact set can be constructed by acting with $G_{2}$ on the 3-plane

$$
\xi_{0}=\left\{\left(x_{1}, x_{2}, x_{3}, 0,0,0,0\right): x_{1}, x_{2}, x_{3} \in \mathbb{R}\right\} \subset \mathbb{R}^{7},
$$

for generalized associative calibrations and on the 4-plane

$$
\xi_{0}=\left\{\left(0,0,0, x_{4}, x_{5}, x_{6}, x_{7}\right): x_{4}, x_{5}, x_{6}, x_{7} \in \mathbb{R}\right\} \subset \mathbb{R}^{7},
$$


for generalized co-associative calibrations.

There are many special cases of seven-dimensional Riemannian manifolds that admit connections whose holonomy is contained in $G_{2}$. We shall present many of these cases when we investigate the deformation theory of generalized associative and co-associative calibrations.

\section{$\underline{\text { Holonomy } \operatorname{Spin}(7)}$}

A Riemannian manifold $(M, g)(\operatorname{dim} M=8)$ equipped with a metric connection $\nabla$ whose holonomy is contained in $\operatorname{Spin}(7)$ admits a $\nabla$-parallel self-dual four-form $\Phi$. As we shall see, $\Phi$ is a generalized Cayley calibration.

Conversely, let $(M, g, \Phi)$ be a Riemannian manifold equipped with a self-dual fourform $\Phi$ which satisfies the algebraic conditions of a $\operatorname{Spin}(7)$ structure, then $(M, g, \Phi)$ admits a connection $\nabla$ whose holonomy is contained in $\operatorname{Spin}(7)$. The connection $\nabla$ can be expressed in terms of the Levi-Civita connection $\nabla^{g}$ of $g$ as

$$
\nabla_{k} Y^{i}=\nabla_{k}^{g} Y^{i}+\frac{1}{96} \Phi^{i}{ }_{m k l} \nabla_{k}^{g} \Phi_{j}{ }^{m k l} Y^{j}
$$

where $Y$ is a vector field.

Proposition 4. Let $(M, g, \Phi)$ be an eight-dimensional manifold with Spin(7) structure as above. The forms $\Phi$ is generalized calibration of degree four. In both cases the contact set at every point of $M$ is $S p i n(7) / K$, where $K=S U(2) \times S U(2) \times$ $S U(2) / Z_{2}$.

Proof. To show this, we remark that there is locally a frame $\left\{e^{A} ; A=1, \ldots, 8\right\}$ of $(M, g, \Phi)$ such that the metric and the $\nabla$-parallel self-dual four-form $\Phi$ can be written as

$$
\begin{aligned}
g & =\sum_{A=1}^{8}\left(e^{A}\right)^{2} \\
\Phi & =e^{1234}+\left(e^{12}-e^{34}\right) \wedge\left(e^{56}-e^{78}\right)+\left(e^{13}+e^{24}\right) \wedge\left(e^{57}+e^{68}\right) \\
& +\left(e^{14}-e^{23}\right) \wedge\left(e^{58}-e^{67}\right)+e^{5678}
\end{aligned}
$$

Observe that in such a frame the metric and parallel self-dual four-form take the standard form of a Euclidean metric and $\operatorname{Spin}(7)$-invariant four-form in $\mathbb{R}^{8}$. It follows that $\Phi$ is a calibration from the results of Harvey and Lawson as they apply for the Cayley calibration. It also follows that the contact set at every point of $M$ is $\operatorname{Spin}(7) / K$, where $K=S U(2) \times S U(2) \times S U(2) / Z_{2}$. All the planes of the contact set can be constructed by acting with $\operatorname{Spin}(7)$ on the 4-plane

$$
\xi_{0}=\left\{\left(x_{1}, x_{2}, x_{3}, x_{4}, 0, \ldots, 0\right): x_{1}, x_{2}, x_{3}, x_{4} \in \mathbb{R}\right\} \subset \mathbb{R}^{8} .
$$

It can be shown that given a self-dual four-form as in (4.33), there is always a connection $\nabla$ with torsion a three-form given in [23] which has holonomy contained in $\operatorname{Spin}(7)$. The torsion is

$$
T=\delta \Phi+\frac{7}{6} *(\theta \wedge \Phi)
$$

where $\theta=\frac{1}{7} *(\delta \Phi \wedge \Phi)$ is the Lee form of the manifold. 
4.3. Useful Formulae. For the investigation of the deformation theory of generalized calibrations, we shall use some formulae which relate the Lie derivative of a form to a covariant derivative. Then

Let $\chi$ be a $\mathrm{k}$-form expressed as $\chi=\frac{1}{k !} \chi_{A_{1} \ldots A_{k}} e^{A_{1}} \wedge \cdots \wedge e^{A_{k}}$ in a frame $\left\{e^{A_{p}}\right\}$.

$$
d \chi=\frac{1}{k !} \nabla_{A_{1}} \chi_{A_{2} \ldots A_{k+1}} e^{A_{1}} \wedge \cdots \wedge e^{A_{k+1}}+\frac{1}{(k-1) !} \chi_{A_{1} \ldots A_{k}} T^{A_{1}} \wedge e^{A_{2}} \wedge \cdots \wedge e^{A_{k}}
$$

where $T^{A}=\nabla e^{A}=\frac{1}{2} T^{A}{ }_{B C} e^{B} \wedge e^{C}$ is the torsion 2-form of $\nabla$ or equivalently

$$
T(X, Y)=\nabla_{X} Y-\nabla_{Y} X-[X, Y]
$$

for vector fields $X, Y$.

The Lie derivative of $\chi$ with respect to a vector field $V=V^{A} e_{A},\left(e^{A}, e_{B}\right)=\delta^{A}{ }_{B}$, is as follows:

$$
\begin{aligned}
& \mathcal{L}_{V} \chi=\left(i_{V} d+d i_{V}\right) \chi=\frac{k+1}{k !} V^{A_{1}}(\nabla \chi)_{A_{1} A_{2} \ldots A_{k+1}} e^{A_{2}} \wedge \cdots \wedge e^{A_{k+1}} \\
& +\frac{1}{(k-1) !} \chi_{A_{1} A_{2} \ldots A_{k}} i_{V} T^{A_{1}} \wedge e^{A_{2}} \wedge \cdots \wedge e^{A_{k}} \\
& +\frac{1}{(k-2) !} V^{B} \chi_{A_{1} B A_{3} \ldots A_{k}} T^{A_{1}} \wedge e^{A_{3}} \wedge \cdots \wedge e^{A_{k}} \\
& +\frac{1}{(k-1) !} \nabla_{A_{1}}\left(V^{B} \chi_{B A_{2} \ldots A_{k}}\right) e^{A_{1}} \wedge e^{A_{2}} \wedge \cdots \wedge e^{A_{k}} \\
& +\frac{1}{(k-2) !} V^{B} \chi_{B A_{2} A_{3} \ldots A_{k}} T^{A_{2}} \wedge e^{A_{3}} \wedge \cdots \wedge e^{A_{k}} \\
& =\frac{1}{k !} V^{B} \nabla_{B} \chi_{A_{1} \ldots A_{k}} e^{A_{1}} \wedge \cdots \wedge e^{A_{k}} \\
& +\frac{1}{(k-1) !} \tilde{\nabla}_{A_{1}} V^{B} \chi_{B A_{2} \ldots A_{k}} e^{A_{1}} \wedge \cdots \wedge e^{A_{k}}
\end{aligned}
$$

where $\tilde{\nabla}$ is the unique connection associated with $\nabla$ which has torsion $\tilde{T}=-T$. To summarize

$$
\mathcal{L}_{V} \chi=\nabla_{V} \chi+\frac{1}{(k-1) !} \chi_{A A_{1} \ldots A_{k-1}} \tilde{\nabla}_{B} V^{A} e^{B} \wedge e^{A_{1}} \wedge \cdots \wedge e^{A_{k-1}}
$$

So, if $\chi$ is $\nabla$-parallel, $\nabla \chi=0$, and

$$
\mathcal{L}_{V} \chi=\frac{1}{(k-1) !} \chi_{A A_{1} \ldots A_{k-1}} \tilde{\nabla}_{B} V^{A} e^{B} \wedge e^{A_{1}} \wedge \cdots \wedge e^{A_{k-1}} .
$$

Another formula which we shall find useful is the Lie derivative of a vector-valued $\mathrm{k}$-form

$$
\xi=\frac{1}{k^{!}} \xi_{A_{1} \ldots A_{k}}{ }^{B} e^{A_{1}} \wedge \cdots \wedge e^{A_{k}} \otimes e_{B}
$$

which may be written as

$$
\begin{aligned}
\mathcal{L}_{V} \xi=\nabla_{V} \xi & +\frac{1}{(k-1) !} \xi_{B A_{2} \ldots A_{k}}{ }^{A} \tilde{\nabla}_{A_{1}} V^{B} e^{A_{1}} \wedge \cdots \wedge e^{A_{k}} \otimes e_{A} \\
& -\frac{1}{k !} \xi_{A_{1} \ldots A_{k}}{ }^{B} \tilde{\nabla}_{B} V^{A} e^{A_{1}} \wedge \cdots \wedge e^{A_{k}} \otimes e_{A}
\end{aligned}
$$


So, if $\xi$ is $\nabla$-parallel, and $X$ is some submanifold on which

$$
\left.\xi_{A_{1} \ldots A_{k}}{ }^{A} e^{A_{1}} \wedge \cdots \wedge e^{A_{k}}\right|_{X} \otimes e_{A}=0
$$

then

$$
\left.\mathcal{L}_{V} \xi\right|_{X}=\left.\frac{1}{(k-1) !} \xi_{B A_{2} \ldots A_{k}}{ }^{A} \tilde{\nabla}_{A_{1}} V^{B} e^{A_{1}} \wedge \cdots \wedge e^{A_{k}}\right|_{X} \otimes e_{A}
$$

These formulae are key in the investigation of the deformation theory of generalized calibrations.

4.4. Second variation of the energy functional revisited. The second variation of the energy functional is considerably simplified if we assume that the calibration form $\phi$ is $\nabla$-parallel. In particular, suppose that $X$ is a k-dimensional submanifold calibrated with respect to $\phi$. Suppose we consider an adapted frame so that $\left\{e^{a}: a=1, \ldots, k\right\}$ are tangent to $X$ and $\left\{e^{i}: i=k+1, \ldots, \operatorname{dim}(\mathrm{M})\right\}$ are normal to $X$, and we take $\left.\phi_{i a_{1} \ldots a_{k-1}}\right|_{t=0} e^{i} \wedge e^{a_{1}} \wedge \cdots \wedge e^{a_{k-1}}=0$. Then we deform the calibration and write the calibration form as

$$
\begin{aligned}
\phi & =\lambda e^{1} \wedge \cdots \wedge e^{k}+\frac{1}{(k-1) !} \phi_{i a_{1} \ldots a_{k-1}} e^{i} \wedge e^{a_{1}} \wedge \cdots \wedge e^{a_{k-1}} \\
& +\frac{1}{(k-2) !} \phi_{i j a_{1} \ldots a_{k-2}} e^{i} \wedge e^{j} \wedge e^{a_{1}} \wedge \cdots \wedge e^{a_{k-2}}+\mathcal{O}\left(\left(e^{i}\right)^{3}\right) .
\end{aligned}
$$

Using the fact that $\phi$ is $\nabla$-parallel, we can compute $\frac{d^{2} \lambda}{d t^{2}}$ by acting twice on (4.46) with $\nabla_{V}$ where $V=\partial / \partial t$ is a normal vector field. After some lengthy computation, we find

$$
\begin{aligned}
\left.\frac{d^{2}}{d t^{2}} E(t)\right|_{t=0} & =-\left.\int_{X} \frac{d^{2}}{d t^{2}} \lambda(t)\right|_{t=0} d \operatorname{vol}(0) \\
& =\left.\int_{X}\left[\left(\tilde{\nabla}^{\perp} V, \tilde{\nabla}^{\perp} V\right) d \operatorname{vol}(0)-i_{\tilde{\nabla}^{\perp} V^{2}} i_{\tilde{\nabla}^{\perp} V} \phi\right]\right|_{t=0} .
\end{aligned}
$$

5. Deformation of hermitian calibrations. Let $(M, g, J)$ be a hermitian manifold of complex dimension $n$. As we have mentioned the calibrated submanifolds with respect to $\phi=\frac{1}{k !} \Omega^{k}$ are the complex submanifolds of $(M, g, J)$ of dimension $k$. The deformation theory of a complex submanifold $X$ of $M$ is well known. The dimension of the moduli space is the number of holomorphic vector fields of the normal bundle of the submanifold $X$, i.e. it is the dimension of the Čech cohomology $\check{\mathrm{H}}^{0}\left(N_{X}\right)$ where $N_{X}$ is the normal bundle of $X$ in $M$. There is an obstruction of integrating these small deformations. This lies in the Čech cohomology group $\check{\mathrm{H}}^{1}\left(N_{X}\right)$. It is clear that there should be a theory of deformations of almost hermitian calibrations. This will be investigated elsewhere.

5.1. Complex submanifolds of Hermitian manifolds. It has been known for some time that hermitian manifolds admit compact complex submanifolds which represent the trivial homology class. This is unlike the case of compact Kähler manifolds where complex submanifolds always represent a non-trivial homology class. An example of such a hermitian manifold that admits a holomorphic submanifold which represents the trivial homology class has been given in [32]. This is an example of a hermitian calibration which is not Kähler. 
Another example of a hermitian calibration is that of the Hopf fibre $S^{1} \times S^{1}$ in the group manifold $S^{3} \times S^{3}$. As we shall demonstrate later in the investigation of examples of SAS calibrations in group manifolds, such a submanifold is holomorphic with respect to a hermitian structure on $S^{3} \times S^{3}$. Observe that the hermitian calibration $S^{1} \times S^{1}$ represents the trivial homology class.

6. Deformations of SAS Calibrations. Let $(M, g, J)$ be an almost complex manifold which admits a compatible connection $\nabla(\nabla g=\nabla J=0)$ which has holonomy contained in $S U(n)$. The following can be shown using the results of Harvey and Lawson:

THEOREM 6. Let $(M, g, J)$ be a manifold as above. If $X$ is a SAS calibrated submanifold of $M$ with respect to $\phi=\operatorname{Re} \omega$, where $\omega$ is the parallel-(n,0) form, then $\left.\Omega\right|_{X}=\left.\operatorname{Im} \omega\right|_{X}=0$, where $\Omega$ is the Hermitian form obtained from $J$. Conversely, if $X$ is a middle dimension submanifold of $M$ such that $\left.\Omega\right|_{X}=\left.\operatorname{Im} \omega\right|_{X}=0$, then $X$ is calibrated with respect to $\phi$.

Because of this, the small deformations of $X$ generated by sections $V$ of the normal bundle, $N_{X}$, of $X$ in $M$ which preserve the property that $X$ is calibrated satisfy

$$
\left.\mathcal{L}_{V} \Omega\right|_{X}=0,\left.\mathcal{L}_{V} \operatorname{Im} \omega\right|_{X}=0 .
$$

To determine the conditions on $V$ imposed by the above two conditions, we proceed as follows. We introduce an orthonormal basis $\left\{e_{a}, e_{a^{\prime}}\right\}=$ $\left\{e_{1}, \ldots, e_{n}, e_{1^{\prime}}, \ldots, e_{n^{\prime}}\right\}$ of the tangent bundle of $M$ and a dual frame $\left\{e^{a}, e^{a^{\prime}}\right\}=$ $\left\{e^{1}, \ldots, e^{n}, e^{1^{\prime}}, \ldots, e^{n^{\prime}}\right\}$ of $M$ such that the Kähler form $\Omega$ and the parallel $(\mathrm{n}, 0)$ form take the (canonical) forms

$$
\begin{aligned}
& \Omega=\sum_{a=1, b^{\prime}=1}^{n} \Omega_{a b^{\prime}} e^{a} \wedge e^{b^{\prime}}=\sum_{a=1, b^{\prime}=1}^{n} \delta_{a b^{\prime}} e^{a} \wedge e^{b^{\prime}}=\sum_{a=1}^{n} e^{a} \wedge e^{a^{\prime}} \\
& \omega=\Pi_{a=1}^{n}\left(e^{a}-i J\left(e^{a}\right)\right)=\left(e^{1}+i e^{1^{\prime}}\right) \wedge \cdots \wedge\left(e^{n}+i e^{n^{\prime}}\right) .
\end{aligned}
$$

It is clear that the non-vanishing components of the almost complex structure $J$ in this frame are $J^{a}{ }_{a^{\prime}}$ and $J^{a^{\prime}}{ }_{a}$.

Restricting the orthonormal basis $\left\{e_{a}, e_{a^{\prime}}\right\}$ at a point $p$ of the calibrated submanifold $X,\left\{e_{a}\right\}$ is a basis in the tangent space $T_{p} X$ and $\left\{e_{a^{\prime}}\right\}$ is a basis in the fibre $N_{p}$ of the normal bundle $N_{X}$ of $X$ in $M$. Therefore the deformations of $X$ in $M$ are described by vector fields $V=V^{a^{\prime}} e_{a^{\prime}}$. Using this and (4.40), the conditions (6.48) can be written as follows:

$$
\begin{aligned}
\tilde{\nabla}_{a} V^{b^{\prime}} \Omega_{b^{\prime} b} e^{a} \wedge e^{b} & =0 \\
\tilde{\nabla}_{a} V^{b^{\prime}} J^{a}{ }_{b^{\prime}} & =0 .
\end{aligned}
$$

These are viewed as equations for the normal vector field $V$.

There is another way to write the deformation equations of SAS calibrations. For this observe that the normal bundle $N_{X}$ and the tangent bundle $T X$ of $X$ are isomorphic, $N_{X} \equiv T X$. The isomorphism is induced by the almost complex structure $J$ as $U=U^{a} e_{a}=J(V)=J^{a}{ }_{b^{\prime}} V^{b^{\prime}} e_{a}$. Using this, the conditions (6.48) on the normal vector field $V$ can be rewritten as

$$
\left.d\left(i_{V} \Omega\right)\right|_{X}+\left.i_{V} d \Omega\right|_{X}=0,\left.d\left(i_{V} \operatorname{Im} \omega\right)\right|_{X}+\left.i_{V} d \operatorname{Im} \omega\right|_{X}=0 .
$$


Next using the fact that both $\Omega$ and $\omega$ are parallel with respect to the connection $\nabla$ and (4.36), these two conditions can be expressed as

$$
D_{1} U \equiv d U-U_{a}\left(T^{a}+\hat{T}^{a}\right)=0
$$

and

$$
D_{0}^{\dagger} U \equiv \delta U+U^{a}\left(t_{a}+\hat{t}_{a}\right)=0,
$$

where $\hat{T}^{a}=e^{b} \wedge e^{c} \Omega_{b b^{\prime}} T^{b^{\prime}}{ }_{c a^{\prime}} \Omega^{a^{\prime} a}, t_{a}=T_{a b}^{b}$ and $\hat{t}_{a}=J^{a^{\prime}}{ }_{a} T^{b^{\prime}}{ }_{a^{\prime} b} J_{b^{\prime}}^{b}$.

Equivalently, the deformation equations (6.52) and (6.53) can be written in components as follows:

$$
\partial_{\mu_{1}} U_{\mu_{2}}-\partial_{\mu_{2}} U_{\mu_{1}}-U_{\rho}\left(T_{\mu_{1} \mu_{2}}^{\rho}+\hat{T}_{\mu_{1} \mu_{2}}^{\rho}\right)=0
$$

and

$$
\left(\nabla^{g}\right)^{\mu} U_{\mu}-U^{\mu}\left(t_{\mu}+\hat{t}_{\mu}\right)=0 .
$$

We remark that the deformations of SAS calibrations in the special case when $M$ is a symplectic manifold, and so $\Omega$ is closed, have been considered in [31]. It has been shown that the moduli are unobstructed, and the dimension of the moduli space is $b_{1}(X)$. Both expressions $(6.50)$ and $(6.52,6.53)$ of the deformation equations will be used later in the examples to find the moduli space of SAS calibrations in nonsymplectic manifolds.

6.1. SAS calibrations and an elliptic system. To investigate whether the differential system (6.52) and (6.53) has solutions, consider the following resolution:

$$
\Lambda^{0}(X) \stackrel{D_{0}}{\rightarrow} \Lambda^{1}(X) \stackrel{D_{1}}{\rightarrow} \Lambda^{2}(X)
$$

where $D_{1}$ has been defined as above and $D_{0}$ is the adjoint of $D_{0}^{\dagger}$. Clearly we have the adjoint resolution as follows:

$$
\Lambda^{0}(X) \stackrel{D_{0}^{\dagger}}{\longleftarrow} \Lambda^{1}(X) \stackrel{D_{1}^{\dagger}}{\longleftarrow} \Lambda^{2}(X),
$$

where $D_{1}^{\dagger}$ is the adjoint of $D_{1}$. Next we can consider the Laplacian

$$
\triangle=D_{0} D_{0}^{\dagger}+D_{1}^{\dagger} D_{1}
$$

From general elliptic theory, we know that the solutions of the equations $D_{1} U=0$ and $D_{0}^{\dagger} U=0$ are zero modes of the Laplacian $\triangle$. Conversely, the zero modes of the Laplacian $\triangle$ are also solutions of the two differential equations. From general elliptic theory we also have the following:

Corollary 4. The moduli space of a closed $S A S$ calibration $X$ in $M$, if it exists, has finite dimension.

We shall investigate the elliptic system in more detail in special cases below.

7. Special Cases. There are several different types of almost hermitian structures, for example given in the Gray-Hervella classification [15]. We shall not explore all cases here. Instead, we shall focus on some of these. Some explicit examples will be given later. In what follows, we shall assume that some hermitian connections have holonomy contained in $S U(n)$. 
7.1. Almost Hermitian manifolds with skew torsion. Let $(M, g, J)$ be an almost hermitian manifold. It has been shown in [10] that $(M, g, J)$ admits a unique almost hermitian connection $\nabla$ with torsion a three-form iff the Nijenhuis tensor of $J$ is a three-form as well, ie $(M, g, J)$ is a $G_{1}$ manifold in the Gray-Hervella classification. In that case the torsion of the connection is

$$
T(X, Y, Z)=-d \Omega(J X, J Y, J Z)+N(X, Y, Z)
$$

where $N(X, Y)=[J X, J Y]-[X, Y]-J[J X, Y]-J[X, J Y]$ is the Nijenhuis tensor. Suppose that in addition the holonomy of this connection is contained in $S U(n)$. In such a case the differential system for SAS calibrations can be simplified to

$$
\begin{aligned}
d U-U_{a}\left(T^{a}+\hat{T}^{a}\right) & =0 \\
\delta U+U^{a} \hat{t}_{a} & =0 .
\end{aligned}
$$

Despite the simplification of the second deformation equation, we have not been able to analyze the system further. However, a special class of such hermitian manifolds are the Nearly Kähler ones. For these, the deformation equations simplify further.

7.2. Nearly Kähler manifolds. Let $(M, g, J)$ be a Nearly Kähler manifold, ie $(M, g, J)$ is an almost hermitian manifold satisfying $\left(\nabla_{X}^{g} J\right) Y+\left(\nabla_{Y}^{g} J\right) X=0$, where $X, Y$ are vector fields on $M$. It is known [14] that on a Nearly Kähler manifold $(M, g, J)$ the following identities hold:

$$
4 d \Omega(X, Y, Z)=3 N(J X, Y, Z)=-12 g\left(\left(\nabla_{X}^{g} J\right) Y, Z\right)
$$

and

$$
2 g\left(\left(\nabla_{U}^{g} \nabla_{X}^{g} J\right) Y, Z\right)=-\left(g\left(\left(\nabla_{U}^{g} J\right) X,\left(\nabla_{Y}^{g} J\right) J Z\right)+\operatorname{cyclic}(X, Y, Z)\right) .
$$

The Nijenhuis tensor $N$ is a $(3,0)+(0,3)$-form. Nearly Kähler manifolds admit a compatible connection $\nabla$ with torsion a three-form $T=\frac{1}{4} N$ and $\nabla T=0[26,4,10]$.

We shall focus our attention to six-dimensional nearly Kähler manifolds. Any six-dimensional nearly Kähler manifold is Einstein and of constant type (see [14]). This means that the Ricci tensor, $R i c^{g}=\frac{5}{2} a g$ and

$$
\left\|\left(\nabla_{X}^{g} J\right) Y\right\|^{2}=\frac{1}{2} a \cdot\left(\|X\|^{2} \cdot\|Y\|^{2}-g^{2}(X, Y)-g^{2}(X, J Y)\right),
$$

where $a=S c_{a l}^{g} / 15$ is a positive constant and $S c^{g} l^{g}$ denotes the scalar curvature of $g$. It is clear that the holonomy of the connection $\nabla$ of any six-dimensional Nearly Kähler manifold which is not Kähler is contained in $S U(3)$.

To investigate SAS calibrations in six-dimensional Nearly Kähler manifolds, we shall first prove the following theorem for Lagrangian submanifolds in Nearly Kähler manifolds.

TheOREM 7. A three-dimensional Lagrangian submanifold $L$ of a six-dimensional Nearly Kähler manifold $(M, J, g)$ is a SAS calibration and minimal. Consequently any Lagrangian submanifold $L$ is orientable.

Proof. To show that any Lagrangian submanifold of a six-dimensional nearly Kähler manifold is minimal, we shall first show that

$$
g\left(\left(\nabla_{X}^{g} J\right) Y, Z\right)=0
$$


for $X, Y, Z$ tangent to $L$. To see this, we use the fact that $J$ is parallel with respect to the connection $\nabla$ with torsion the Nijenhuis tensor $N$. Using the fact that $N$ is a $(3,0)+(0,3)$-form and that $L$ is Lagrangian, it is straightforward to verify (7.64).

To show that $L$ is SAS, observe that a $\nabla$-parallel $(3,0)$ form $\psi$ can be defined on $L$ with $\operatorname{Re} \psi=N$ and $\operatorname{Im} \psi=\frac{3}{4} d \Omega ; \Omega$ is the Kähler form. Since both the Nijenhuis tensor $N$ and the almost complex structure $J$ are $\nabla$-parallel, in view of (7.61), $\psi$ is parallel as well. So $N$ can be identified with the calibration form. Using (7.64), $\left.(d \Omega)\right|_{L}=0$ and so $L$ is a SAS calibration. Consequently $L$ is orientable.

The second part of the proof of this theorem that $L$ is minimal is a generalization of a similar theorem for $S^{6}$ in [8]. To begin, denote with $\alpha$ and $A$ the second fundamental form and the shape operator of the submanifold $L$ in a manifold $M$, respectively. From the definition of $\alpha$ and $A$, we have $X, Y$ tangent to $L$ and $\xi$ normal to $L$

$$
\nabla_{X}^{g} Y=\nabla_{X}^{g_{L}} Y+\alpha(X, Y), \quad \nabla_{X}^{g} \xi=-A_{\xi} X+D_{X} \xi
$$

where $X, Y$ are vector fields tangent to $L, \xi$ is a vector field normal to $L, \nabla^{g_{L}}$ is the induced Levi-Civita connection on $L$ and $D$ is the induced connection on the normal bundle. Recall that $\alpha(X, Y)=\alpha(Y, X)$ and $g(\alpha(X, Y), \xi)=g\left(A_{\xi} X, Y\right)$.

To see that $L$ is minimal we observe that the $(3,0)+(0,3)$-form $d \Omega$ on $M$ satisfies the identity

$$
-\frac{1}{3}\left(\nabla_{X}^{g} d \Omega\right)(Y, Z)=\left(\nabla_{X}^{g} \nabla_{Y}^{g} J\right) Z=\frac{1}{2} a(g(Y, J Z) X+g(X, Z) J Y-g(X, Y) J Z),
$$

where $d \Omega(X, Y)$ denotes the $(1,2)$ tensor corresponding to the 3 -form $d \Omega$ via the metric $g$.

Since $d \Omega(X, Y)$ is normal to $L$ for $X, Y$ tangent to $L$, we obtain $D_{X} J Y=$ $-\frac{1}{3} d \Omega(X, Y)+J \nabla_{X}^{g_{L}} Y, \quad A_{J Y} X=-J \alpha(X, Y)$. Using these properties of the second fundamental form, we calculate

$$
\begin{aligned}
\left(\nabla_{X}^{g} d \Omega\right)(Y, Z)= & -A_{d \Omega(Y, Z)} X+D_{X} d \Omega(Y, Z)-d \Omega\left(\nabla_{X}^{g} Y, Z\right)-d \Omega\left(Y, \nabla_{X}^{g} Z\right) \\
= & J \alpha(J d \Omega(Y, Z), X)-\frac{1}{3} J d \Omega(X, d \Omega(Y, Z))-J\left(\nabla_{X}^{g_{L}} J d \Omega\right)(Y, Z) \\
& -d \Omega(\alpha(X, Y), Z)-d \Omega(Y, \alpha(X, Z))
\end{aligned}
$$

for $X, Y, Z$ tangent to $L$. Multiplying the last equality by $J$ and using (7.66) we get for the normal component

$$
\alpha(J d \Omega(Y, Z), X)+J d \Omega(\alpha(X, Y), Z)+J d \Omega(Y, \alpha(X, Z))=0 .
$$

The last equality means $\operatorname{tr} \alpha=0$. Indeed, we may assume that $\frac{1}{3} J d \Omega\left(e_{1}, e_{2}\right)=\sqrt{\frac{a}{2}} \cdot e_{3}$ form an orthonormal basis on $L$ for any even permutation of (123). Evaluating (7.68) on those basis we get $\operatorname{tr} \alpha=0$ by taking the cyclic sum and using the skew-symmetry of $N$. Hence, $L$ is a minimal submanifold of $M$.

The theorem above generalizes the result of Ejiri [8] which states that for the Nearly Kähler $S^{6}$ any Lagrangian submanifold $M^{3} \subset S^{6}$ is minimal. SAS calibrations in the Nearly Kähler $S^{6}$ will be consider below.

Since the torsion is a $(3,0)+(0,3)$ form, the differential system for the deformation of SAS calibrations on a nearly Kähler six-dimensional manifold $(M, J, g)$ reduces to the equations

$$
d U=\frac{3}{4}\left(i_{U}\right) N=3\left(i_{U} T\right)=-\left(i_{J U}\right) d \Omega, \quad \delta U=0
$$


Proposition 5. Let $V=J U$ be a SAS deformation of a SAS calibrated compact submanifold $L$ on 6-dimensional Nearly Kähler manifold $M$. Then the following formula holds

$$
\int_{L}\left(2 R i c^{g}(U, U)-\frac{9}{2} a\|U\|^{2}+\frac{1}{2}\left(\mathcal{L}_{U} g\right)^{2}\right) d V o l .\left.\right|_{L}=0 .
$$

In particular, if $M=S^{6}$ then $U$ cannot be a Killing vector field on $L$.

Proof. We shall use the following general formula on a compact Riemannian manifold [34]

$$
\int_{L}\left(R i c_{i j} U^{i} U^{j}+\left(\nabla^{j} U^{i}\right)\left(\nabla_{i} U_{j}\right)-(\delta U)^{2}\right) d V o l .=0
$$

The formula (7.71) follows from the identity

$$
\nabla_{i}\left(U^{j}\left(\nabla_{j} U^{i}\right)-\left(\nabla_{j} U^{j}\right) U^{i}\right)=R i c_{i j} U^{i} U^{j}+\left(\nabla^{j} U^{i}\right)\left(\nabla_{i} U_{j}\right)-\left(\nabla_{j} U^{j}\right)^{2}
$$

by an integration over the compact $L$.

Let $V=J U$ be a SAS deformation. The constant type condition (7.63) implies $\|d U\|^{2}=9\left\|i_{U} T\right\|^{2}=9 a\|U\|^{2}$. Substituting the latter equality into (7.71) we get $(7.70)$.

For any minimal lagrangian submanifold $L$ of the Nearly Kähler $S^{6}$ we have $\operatorname{Ric}^{g}(U, U)=a\|U\|^{2}-\sum_{i=1}^{3} g\left(\alpha\left(U, e_{i}\right), \alpha\left(U, e_{i}\right)\right)$, where $e_{1}, e_{2}, e_{3}$ is an orthonormal basis on $L$. Substituting the last equality into (7.70) and taking into account (7.69), we get a contradiction with the assumption that $U$ is Killing.

7.3. Hermitian manifolds with holonomy $S U(n)$. In this section, we take $(M, J, g)$ to be a hermitian manifold, $\operatorname{dim} M=2 n$, for which the holonomy of either the Bismut connection $\nabla^{b}$ or the Chern connection $\nabla^{c}$ is contained in $S U(n)$. The definitions of these connections are given in section fifteen. Both these cases will emerge in the investigation of hermitian manifolds with trivial canonical bundle in sections sixteen and seventeen.

First consider the case for which the Bismut connection has holonomy contained in $S U(n)$. In such a case the differential system for the deformation of SAS calibrations becomes

$$
\begin{aligned}
d U-\frac{1}{2} U^{a} H_{a b^{\prime} c^{\prime}} J^{b^{\prime}}{ }_{b} J^{c^{\prime}}{ }_{c} e^{b} \wedge e^{c} & =0 \\
\left(\nabla^{g}\right)^{a} U_{a}-U^{a} \theta_{a} & =0
\end{aligned}
$$

where $H$ is the torsion of the Bismut connection and $\theta$ is the Lee form (see section fifteen). To derive the first equation, we have used the fact that the torsion three-form $H$ of the Bismut connection is $(2,1)$ and $(1,2)$ with respect to $J$; this follows from the integrability of the complex structure and the fact that $J$ is parallel with respect to the Bismut connection.

There are two cases to consider. If the hermitian manifold $(M, g, J)$ is balanced, then $\theta=0$, and the deformation equations are

$$
\begin{aligned}
d U-\frac{1}{2} U^{a} H_{a b^{\prime} c^{\prime}} J^{b^{\prime}}{ }_{b} J^{c^{\prime}}{ }_{c} e^{b} \wedge e^{c} & =0 \\
\left(\nabla^{g}\right)^{a} U_{a} & =0 .
\end{aligned}
$$


In particular, $U$ is co-closed. Next assume that $(M, g, J)$ is conformally balanced, ie $\theta=2 d f$ for some function $f$ on $M$. This class of hermitian structures appears in some applications in physics. Rescaling $U=e^{2 f} \hat{U}$, we find that the differential system becomes

$$
\begin{aligned}
d \hat{U}+2 d f \wedge \hat{U}-\frac{1}{2} \hat{U}^{a} H_{a b^{\prime} c^{\prime}} J^{b^{\prime}}{ }_{b}{ }^{c^{\prime}}{ }_{c} e^{b} \wedge e^{c} & =0 \\
\left(\nabla^{g}\right)^{a} \hat{U}_{a} & =0 .
\end{aligned}
$$

So $\hat{U}$ is again co-closed.

Next take $(M, J, g)$ to be a hermitian manifold for which the associated Chern connection $\nabla^{c}$ has holonomy contained in $S U(n)$. In this case the parallel $(\mathrm{n}, 0)$-form $\psi$ is holomorphic and therefore closed, $d \psi=0$. The deformation differential system $(6.54,6.55)$ for SAS calibrations becomes

$$
\begin{aligned}
d U-U_{a}\left(C^{a}+\hat{C}^{a}\right) & =0 \\
\left(\nabla^{g}\right)^{a} U_{a} & =0
\end{aligned}
$$

where $C$ is the torsion of the Chern connection. Again, the one-form $U$ is co-closed.

\section{Examples.}

SAS calibrations in hermitian group manifolds metric

8.1. Group manifold examples. Consider the group manifold $S^{3} \times S^{3}$ with

$$
d s^{2}=\left(\sigma^{1}\right)^{2}+\left(\sigma^{2}\right)^{2}+\left(\sigma^{3}\right)^{2}+\left(\tilde{\sigma}^{1}\right)^{2}+\left(\tilde{\sigma}^{2}\right)^{2}+\left(\tilde{\sigma}^{3}\right)^{2}
$$

and a complex structure $J$ with associated Kähler form

$$
\Omega=\sigma^{1} \wedge \sigma^{2}-\tilde{\sigma}^{1} \wedge \tilde{\sigma}^{2}+\sigma^{3} \wedge \tilde{\sigma}^{3}
$$

where $\sigma^{i}$ and $\tilde{\sigma}^{i}$ are left invariant one-forms satisfying

$$
\begin{aligned}
& d \sigma^{a}=-\frac{1}{2} \epsilon^{a b c} \sigma^{b} \wedge \sigma^{c} \\
& d \tilde{\sigma}^{a}=-\frac{1}{2} \epsilon^{a b c} \tilde{\sigma}^{b} \wedge \tilde{\sigma}^{c} .
\end{aligned}
$$

The associated parallel $(3,0)$-form is

$$
\omega=e^{i \frac{\pi}{4}}\left(\sigma^{1}+i \sigma^{2}\right) \wedge\left(\tilde{\sigma}^{1}-i \tilde{\sigma}^{2}\right) \wedge\left(\sigma^{3}+i \tilde{\sigma}^{3}\right)
$$

The $X=S^{3}$ submanifold of $S^{3} \times S^{3}$ which is defined by the diagonal embedding $\left.\sigma^{i}\right|_{X}=\left.\tilde{\sigma}^{i}\right|_{X}$ is a SAS calibration.

This SAS calibration has moduli. To see this observe that both $\Omega$ and $\omega$ are invariant under the left action of $S^{3} \times S^{3}$. In addition $S^{3} \times S^{3}$ acts on the diagonal $S^{3}$ as $\left(k_{1}, k_{2}\right)(g, g) \rightarrow\left(k_{1} g, k_{2} g\right)$. Thus if the diagonal $S^{3}$ is a SAS calibration, then all the right cosets of $S^{3}$ in $S^{3} \times S^{3}$ are SAS calibrations as well. The moduli space of these deformations is $S^{3} \times S^{3} / S^{3}$. Observe that if $\left(k_{1}, k_{2}\right)=\left(k_{1}^{\prime} h, k_{2}^{\prime} h\right)$ for $h \in S^{3}$, then $\left(k_{1}, k_{2}\right)$ and $\left(k_{1}^{\prime}, k_{2}^{\prime}\right)$ generate the same deformation. 
For an alternative way to see this, let $\nabla$ be the connection on the group $S^{3} \times S^{3}$ associated with the left action. Observe that the metric and the Kähler form are parallel with respect to the connection $\nabla$. The connection $\tilde{\nabla}$ which has torsion $\tilde{T}=$ $-T$ is associated with the right action on the group manifold $S^{3} \times S^{3}$. In particular all right-invariant vector fields are parallel with respect to $\tilde{\nabla}$. Thus they satisfy the equation (6.50). This is equivalent to the analysis above in which the left group action was used. This is because the right-invariant vector fields generate the left-action on group manifolds. Of course the right-invariant vector fields which are tangent to the diagonal $S^{3}$ generate diffeomorphisms of the diagonal $S^{3}$ and so they are not tangent to the moduli space. However, there are three linearly independent right invariant vector fields which are normal to the diagonal $S^{3}$, which are given by

$$
V_{(i)}^{(R)}=\rho_{i}-\tilde{\rho}_{i}
$$

for $i=1,2,3$ where $\left\{\rho_{i}, \tilde{\rho}_{i} ; i=1,2,3\right\}$ are right invariant vector fields on $S^{3} \times S^{3}$. Hence the dimension of the moduli space is at least three.

To find whether the moduli space has dimension more than three, one should find the number of solutions to the differential equations (6.52 and (6.53) or equivalently (6.50). Adapting them to this example, we have

$$
\begin{array}{r}
d U-U \wedge \sigma^{3}+U_{3} \sigma^{1} \wedge \sigma^{2}=0 \\
\delta U-U_{3}=0
\end{array}
$$

where $\left.U \equiv U_{i} \sigma^{i}\right|_{X}$. After some computation, it can be shown that the only solutions to $(8.82)$ are given by linear combinations of $\left.i_{V_{(i)}^{(R)}} \Omega\right|_{X}$ as expected. Hence the moduli space is three-dimensional.

We remark that there is a hermitian calibration which is a torus $T^{2}=S^{1} \times S^{1}$ along the directions $\left(\sigma^{3}, \tilde{\sigma}^{3}\right)$ of $S^{3} \times S^{3}$. This torus is the fiber of the product of fibers of the product Hopf fibration $T^{2} \rightarrow S^{3} \times S^{3} \rightarrow S^{2} \times S^{2}$. The homology class $\left[T^{2}\right]$ is trivial because $H_{2}\left(S^{3} \times S^{3}\right)=0$. This is an example of a family of hermitian calibrations with base space $S^{2} \times S^{2}$.

A SAS calibration on the complex Iwasawa manifold is given at the end of the paper, in the last section.

\section{SAS calibrations in almost hermitian group manifolds}

For another group manifold example consider again the group manifold $S^{3} \times$ $S^{3}$ with metric (8.77) but now equipped with the almost complex structure $J$ with associated Kähler form

$$
\Omega=\sigma^{1} \wedge \tilde{\sigma}^{1}+\sigma^{2} \wedge \tilde{\sigma}^{2}+\sigma^{3} \wedge \tilde{\sigma}^{3}
$$

The associated $(3,0)$-form is

$$
\omega=\left(\sigma^{1}+i \tilde{\sigma}^{1}\right) \wedge\left(\sigma^{2}+i \tilde{\sigma}^{2}\right) \wedge\left(\sigma^{3}+i \tilde{\sigma}^{3}\right) .
$$

It is clear that the three-sphere given by $S^{3} \times\{e\}$ is a SAS cycle, where $e$ is the identity element. It is also clear that any three-sphere in $S^{3} \times S^{3}$ given by the embedding $S^{3} \times\{k\}, k \in S^{3}$, is again a SAS cycle for the above generalized calibration. Thus there is a moduli space which has dimension at least three. This can also be derived using the connection $\tilde{\nabla}$ as in the other example above. In fact the dimension of the 
moduli space is three. To see this observe that the deformation equations for SAS calibrations imply that

$$
\begin{aligned}
\nabla_{a} U_{b}-\nabla_{b} U_{a} & =0 \\
\nabla_{a} U^{a} & =0,
\end{aligned}
$$

where $\nabla$ is the flat connection on $S^{3}$ with associated frame the left-invariant 1-forms $\left\{\sigma^{a} ; a=1,2,3\right\}$. These equations in particular imply that

$$
\nabla^{a} \nabla_{a} U_{b}=0 \text {. }
$$

Using

$$
\int_{S^{3}}\|\nabla U\|^{2}=-\int_{S^{3}}\left(U, \nabla^{2} U\right)=0
$$

we conclude that $U$ is parallel with respect to $\nabla$ and so left-invariant. Since there are three linearly independent left-invariant vector fields on $S^{3}$, the dimension of the moduli space is three. In fact the moduli space is $S^{3}$.

We can also consider a similar group manifold example as above but this time with

$$
\omega=i\left(\sigma^{1}+i \tilde{\sigma}^{1}\right) \wedge\left(\sigma^{2}+i \tilde{\sigma}^{2}\right) \wedge\left(\sigma^{2}+i \tilde{\sigma}^{2}\right)
$$

as a $(3,0)$ form. In this case a SAS cycle is $\{e\} \times S^{3}$. The moduli space is again $S^{3}$.

We remark that in both the above group manifold examples, there is an almost hermitian calibration which is a torus $T^{2}$ along the directions $\left(\sigma^{3}, \tilde{\sigma}^{3}\right)$. In fact the induced almost complex structure on $T^{2}$ is integrable and so $T^{2}$ is complex.

The above two group manifold examples can be easily generalized as follows. Let $G$ be a semisimple Lie group $(\operatorname{dim} G=k)$. On the group manifold $G \times G$, we can define the metric

$$
d s^{2}(G \times G)=\sum_{a=1}^{k}\left(\left(\sigma^{a}\right)^{2}+\left(\tilde{\sigma}^{a}\right)^{2}\right)
$$

and the almost complex structure $J$ with associated Kähler form

$$
\Omega=\sum_{a=1}^{k} \sigma^{a} \wedge \tilde{\sigma}^{a}
$$

where $\left\{\sigma^{a} ; a=1, \ldots, k\right\}$ and $\left\{\tilde{\sigma}^{a} ; a=1, \ldots, k\right\}$ are the left invariant one-forms of $G \times G$; the first set is that of the first group in the product $G \times G$ while the second set is that of the second group. We can also define a $(\mathrm{n}, 0)$-form as

$$
\omega=\left(\sigma^{1}+i \tilde{\sigma}^{1}\right) \wedge \cdots \wedge\left(\sigma^{k}+i \tilde{\sigma}^{k}\right) .
$$

The submanifold $G \times\{e\}$ is a SAS calibration with respect to $\operatorname{Re} \omega$. In fact all spaces $G \times\{h\}, h \in G$, are SAS calibrations. Therefore the dimension of the moduli space is at least $k$. In fact it can be shown that the dimension of the moduli space is exactly $k$ by repeating the analysis for $S^{3} \times S^{3}$ examples above. In particular, it is straightforward to show that the solutions of the deformation equations $U=-\left.i_{V} \Omega\right|_{X}$ are left-invariant one-forms. Similarly $\{h\} \times G$ are also SAS calibrations with respect to $\operatorname{Re} \omega$ where

$$
\omega=(i)^{-k}\left(\sigma^{1}+i \tilde{\sigma}^{1}\right) \wedge \cdots \wedge\left(\sigma^{k}+i \tilde{\sigma}^{k}\right)
$$

in this case. The dimension of the moduli space is again $k$. 
8.2. SAS submanifolds in $S^{3} \times S^{3}$ with the left-invariant Einstein metric. There is another Nearly Kähler structure on $S^{3} \times S^{3}$ which can be constructed as follows. First write $\operatorname{Spin}(4)=S^{3} \times S^{3}$ and decompose the Lie algebra of $\operatorname{Spin}(4)$ as $\operatorname{spin}(4)=\operatorname{so}(4)=h+m$ with $h=\operatorname{span}\left\{E_{12}, E_{13}, E_{23}\right\}$ and $m=\operatorname{span}\left\{E_{14}, E_{24}, E_{34}\right\}$, where the matrices $E_{i j},(i<j)$ are the standard generators of $s o(4)$. Denoting the associated left-invariant forms as the elements of the basis, we have

$$
d E_{i j}=-\sum_{k=1}^{4} E_{i k} \wedge E_{k j} .
$$

Denote the Killing form on $\operatorname{spin}(4)$ by $B(X, Y)=-1 / 2 \operatorname{tr}(X Y)$. Then there are two Einstein metrics on $S^{3} \times S^{3}$. One is associated with the bi-invariant metric $B_{1}=\left.B\right|_{h \times h}+\left.B\right|_{m \times m}$. The SAS calibrations for this manifold have already been investigated above. The other is associated with the left-invariant metric $B_{1 / 3}=$ $\left.\frac{1}{3} B\right|_{h \times h}+\left.B\right|_{m \times m}$. With respect to $B_{1 / 3}$ we consider the orthonormal basis $e_{1}=$ $\sqrt{3} E_{12}, e_{2}=\sqrt{3} E_{13}, e_{3}=\sqrt{3} E_{23}, e_{4}=E_{14}, e_{5}=E_{24}, e_{6}=E_{34}$. In this basis the Kähler form is

$$
\Omega=-\frac{1}{2}\left(e^{1} \wedge e^{6}-e^{2} \wedge e^{5}+e^{3} \wedge e^{4}\right) .
$$

Denote the associated almost complex structure with $J$. Then $\left(S^{3} \times S^{3}, B_{1 / 3}, J\right)$ is a Nearly Kähler non-Kähler manifold [16].

Consider a copy of $S^{3} \subset S^{3} \times S^{3}$ determined by the integrable distribution $h=$ $\operatorname{span}\left\{e_{1}, e_{2}, e_{3}\right\}$. This is a lagrangian submanifold of the Nearly Kähler manifold $\left(S^{3} \times S^{3}, B_{1 / 3}, J\right)$ and therefore it is a SAS calibration.

The moduli space is at least 3-dimensional. Indeed, simple calculations show that

$$
d e^{j}=-\left(i_{J e_{j}} d \Omega\right), \quad \delta e^{j}=0
$$

for $j=1,2,3$, where $e^{j}$ is the dual 1-form to $e_{j}$. Hence, $e_{1}, e_{2}, e_{3}$ are solutions of the differential system (7.69).

8.3. SAS calibrations on Flag manifold. Let $F_{1,2}=\mathbf{U}(3) / \mathbf{U}(1) \times \mathbf{U}(1) \times$ $\mathrm{U}(1)$ be the complex three-dimensional flag manifold. Consider the reductive decomposition $\mathbf{u}(3)=\mathbf{h} \oplus \mathbf{m}$ where $\mathbf{u}(3)$ is the Lie algebra of the unitary group $\mathbf{U}(3)$ and $\mathbf{h}$ and $\mathbf{m}$ are determined by: $\mathbf{h}=\cong \mathbf{u}(1) \oplus \mathbf{u}(1) \oplus \mathbf{u}(1) \subset \mathbf{u}(3)$ and

$$
\mathbf{m}=\left\{\begin{array}{lll}
0 & a & b \\
-\bar{a} & 0 & c \\
-\bar{b} & -\bar{c} & 0
\end{array}\right\} \subset \mathbf{u}(3) .
$$

Identifying any element $X \in T F_{1,2} \cong \mathrm{m}$ with the corresponding triple of complex numbers $(a, b, c)$, we consider the $\mathbf{U}(3)$-invariant Riemannian metric on $F_{1,2}, g(X, X)=|a|^{2}+|b|^{2}+|c|^{2}$. An invariant almost complex structure on $F_{1,2}$ is defined by $J:(a, b, c) \rightarrow(i a,-i b, i c)$ and it is compatible with the invariant metric $g$. Then $\left(F_{1,2}, g, J\right)$ is a Nearly Kähler non Kähler 6-dimensional manifold. We consider an orthonormal basis of $T F_{1,2}$ given by

$$
\begin{array}{r}
e_{1}=\frac{1}{\sqrt{2}}(1,0,0), \quad e_{2}=J e_{1}, \quad e_{3}=\frac{1}{\sqrt{2}}(0,1,0), \\
e_{4}=-J e_{3}, \quad e_{5}=\frac{1}{\sqrt{2}}(0,0,1), \quad e_{6}=J e_{5} .
\end{array}
$$


Then the 3 -sphere $S^{3}$ determined by the integrable lagrangian distribution $e_{1}, e_{3}, e_{5}$ is a SAS calibration on the Nearly Kähler 6-dimensional $\left(F_{1,2}, g, J\right)$.

8.4. SAS calibrations and the 6-sphere. Let $\operatorname{Im\mathcal {O}}$ be the 7-dimensional vector space of imaginary octonions. Consider the unit sphere $S^{6} \subset \operatorname{ImO}$. The right multiplication by $u \in S^{6}$ induces a linear transformation $J_{u}: \mathcal{O} \rightarrow \mathcal{O}$ which is orthogonal and satisfies $J^{2}=-1$. The operator $J_{u}$ preserves the 2-plane spanned by 1 and $u$ and therefore preserves its orthogonal 6-plane which may be identified with $T_{u} S^{6}$. Thus $J_{u}$ induces an almost complex structure on $T_{u} S^{6}$ which is compatible with the inner product induced by the inner product of $\mathcal{O}$. Therefore $S^{6}$ has an almost complex structure which is compatible with the standard metric $g$ on $S^{6}$ and so $\left(S^{6}, g, J\right)$ is an almost hermitian manifold. In fact $\left(S^{6}, g, J\right)$ is Nearly Kähler. The group of automorphisms is the exceptional group $G_{2}$.

Let $L \subset S^{6}$ be a three-dimensional Lagrangian submanifold of $S^{6}$ with respect to a Kähler 2-form. Then by the results of section $7.2, L$ is a SAS calibration and is minimal. In addition it satisfies $\left.g\left(\nabla_{X}^{g} J\right) Y, Z\right)=0$ for $X, Y, Z$ tangent vectors to $L$ by the result of N.Ejiri [8]. For example consider the invariant $G_{2}$ form in (4.29) or equivalently in (9.99) below and view $\left\{e^{i} ; i=1, \ldots, 7\right\}$ an orthonormal basis in $\mathbb{R}^{7}$. Then the Kähler form at the point $x=x^{i} e_{i}$ of $S^{6}, \sum_{i=1}^{7}\left(x^{i}\right)^{2}=1$, is

$$
\Omega=x^{i} \phi_{i j k} e^{j} \wedge e^{k}
$$

restricted in the directions orthogonal to $x$. The three-sphere defined by the equations $x^{1}=x^{2}=x^{3}=0$ is a Lagrangian submanifold and so a SAS calibration.

9. Deformations of generalized co-associative calibrations. Let $(M, g, \psi)$ be a seven-dimensional manifold which admits a metric connection $\nabla$ whose holonomy is contained in $G_{2}$. As we have mentioned there is a local orthonormal frame $\left\{e^{a}, e^{i}\right\}$ for $a, b=4,5,6,7$ and $i, j=1,2,3$ such that the parallel three-form $\psi$ takes the canonical form

$$
\psi=e^{123}+e^{1} \wedge\left(e^{45}-e^{67}\right)+e^{2} \wedge\left(e^{46}+e^{57}\right)+e^{3} \wedge\left(e^{47}-e^{56}\right),
$$

where $e^{12}=e^{1} \wedge e^{2}$ and similarly for the rest. Observe that $\psi$ can also be written as

$$
\psi=e^{123}+\sum_{i=1}^{3} e^{i} \wedge \Omega_{i}
$$

where $\left\{\Omega_{i}, i=1,2,3\right\}$ is a basis of anti-self-dual two-forms in the directions spanned by the $\left\{e^{a} ; a=4,5,6,7\right\}$ frame basis. The generalized co-associative calibrating fourform is simply the Hodge dual of $\psi, * \psi$.

Proposition 6. A necessary and sufficient condition for a four-dimensional submanifold $X$ of $M$ to be a generalized co-associative calibration with respect to $* \psi$ is that $\left.\psi\right|_{X}=0$.

Proof. The proof of this proposition is similar to that given for standard coassociative calibrations in [19] and so it will not be repeated here.

If $X$ is a co-associative calibrated submanifold, we can adapt a frame at every point of $X$ such that the directions $\left\{e^{a} ; a=4,5,6,7\right\}$ are tangent to $X$ and $\left\{e^{i} ; i=\right.$ $1,2,3\}$ are normal. Expressing the condition $\mathcal{L}_{V} \psi=0$ for the deformation of a coassociative calibration $X$ along the normal vector field $V$ in terms of the $\tilde{\nabla}$ connection of $(4.40)$, we have

$$
\left(\Omega_{i}\right)_{a b} \tilde{\nabla}_{c} V^{i} e^{a} \wedge e^{b} \wedge e^{c}=0
$$


where $\left\{\Omega_{i} ; i=1,2,3\right\}$ is the basis of anti-self-dual Kähler forms used to construct the form $\psi$ in (9.99). Using the anti-self duality of $\left\{\Omega_{i} ; i=1,2,3\right\}$, the equation (9.101) also implies

$$
\sum_{i, a}\left(J_{i}\right)_{b}^{a} \tilde{\nabla}_{a} V^{i}=0
$$

where $J_{i}$ are the (almost) complex structures associated with $\Omega_{i}$.

There is an alternative way to express the deformation equations. For this observe that the normal bundle $N_{X}$ of $X$ in $M$ is isomorphic to the bundle $\Lambda^{2-}(X)$ of antiself-dual two-forms of $X, N_{X}=\Lambda^{2-}(X)$. The proof of this is similar to that given by [29]. It is based on the observation that the normal bundle $N_{X}$ and $\Lambda^{2-}(X)$ are both associated to the principal $S O(4)$ frame bundle of $X$ with the same representation, i.e. the three-dimensional anti-self-dual representation of $S O(4)$. Note that the sevendimensional $\rho_{7}$ representation of $G_{2}$ which leaves three form $\psi$ invariant decomposes as $\rho_{7}=v_{3} \oplus v_{4}$ under the action of $S O(4) \subset G_{2}$, where $v_{3}$ is the three-dimensional antiself-dual representation of $S O(4)$ acting on the directions 123 and $v_{4}$ is the standard four-dimensional vector representation of $S O(4)$ acting on the directions 4567 . Let $V=V^{i} e_{i}$ be a normal vector field of $X$, then the isomorphism is given by $\alpha_{V}=\left.i_{V} \psi\right|_{X}$. Observe that $\alpha_{V}$ is an anti-self-dual two-form on $X$.

Next the condition $\mathcal{L}_{V} \psi=0$ can be written as

$$
d \alpha_{V}+\left.i_{V} d \psi\right|_{X}=0 .
$$

If $d \psi=0$, then the dimension of the moduli space is equal to $b_{-}^{2}(X)$, i.e. the dimension of the space of anti-self-dual harmonic two-forms of $X$. Now we shall turn to the case where $d \psi \neq 0$. Using the fact that $\psi$ is parallel with respect to $\nabla$, we find that

$$
d\left(\alpha_{V}\right)-\left(\alpha_{V}\right)_{a b} T^{a} \wedge e^{b}+\frac{1}{2} \psi_{i b c} V^{j} T^{i}{ }_{j a} e^{a} \wedge e^{b} \wedge e^{c}=0
$$

Using the relation

$$
g_{A B}=\frac{1}{6} \psi_{A C D} \psi_{B}^{C D},
$$

the deformation equations may be written solely in terms of $\alpha_{V}$ as

$$
d \alpha_{V}+\left(\alpha_{V}\right)_{a b}\left[-T^{a} \wedge e^{b}+\frac{1}{6} \psi_{i c d} \psi^{j a b} T^{i}{ }_{j a_{1}} e^{c} \wedge e^{d} \wedge e^{a_{1}}\right]=0
$$

Furthermore, as $\alpha_{V}$ is anti-self-dual, this defines an elliptic system of partial differential equations. Hence we conclude that

COROLlARY 5. The moduli space of generalized co-associative calibrations, if it exists, is finite dimensional.

10. Deformations of generalized associative calibrations. Let $(M, g, \psi)$ be a seven-dimensional manifold which admits a metric connection $\nabla$ whose holonomy is contained in $G_{2}$. Such manifolds also admit generalized associative calibrations, in addition to the generalized co-associative calibrations investigated in the previous section. The former is a degree three calibration associated with the three form $\psi$ of (9.99). We again introduce the orthonormal frame $\left\{e^{A}\right\}=\left\{e^{i}, e^{a} ; i=1,2,3, a=\right.$ $4,5,6,7\}$, where now $\left\{e^{i}\right\}$ span the tangent directions of the generalized associative cycle and $\left\{e^{a}\right\}$ span the normal directions. 
As in the case of standard calibrations, the condition for a three-dimensional submanifold $X$ to be calibrated with respect to $\psi$ is that a certain vector-valued three-form $\chi \in \Omega^{3}(M, T M)$ should vanish on $X$. The form $\chi$ is related to the cross product on $\mathbb{R}^{7}=\operatorname{Im} \mathbb{O}$ and it is invariant under $G_{2}$, so $\chi$ is $\nabla$-parallel. In particular in the basis that we have written the three-form $\psi, \chi$ is given by

$$
\chi=\sum_{A=1}^{7} \chi^{A} \otimes e_{A}
$$

where

$$
\begin{aligned}
& \chi^{1}=\left(e^{256}-e^{247}+e^{346}+e^{357}\right) \\
& \chi^{2}=\left(e^{147}-e^{156}-e^{345}+e^{367}\right) \\
& \chi^{3}=\left(e^{245}-e^{267}-e^{146}-e^{157}\right) \\
& \chi^{4}=\left(e^{567}-e^{127}+e^{136}-e^{235}\right) \\
& \chi^{5}=\left(e^{126}-e^{467}+e^{137}+e^{234}\right) \\
& \chi^{6}=\left(e^{457}-e^{125}-e^{134}+e^{237}\right) \\
& \chi^{7}=\left(e^{124}-e^{456}-e^{135}-e^{236}\right) .
\end{aligned}
$$

To compute the dimension of the moduli space of generalized associative calibrations, we require that $\left.\mathcal{L}_{V} \chi\right|_{X}=0$. Using the fact that this vector-valued threeform is parallel with respect to the connection $\nabla,(4.40)$ and after observing that $\left.\left(\chi^{A} \mathcal{L}_{V} e_{A}\right)\right|_{X}=0$, we find that $\left.\mathcal{L}_{V} \chi\right|_{X}=0$ implies

$$
\sum_{i, b}\left(\Omega_{i}\right)_{a b} \tilde{\nabla}_{i} V^{b}=0
$$

where $\tilde{\nabla}$ is the connection with torsion $\tilde{T}=-T$. The normal bundle $N_{X}$ of a generalized associative submanifold is isomorphic to the spin bundle $\mathbb{S}$ of $X$. This can be shown by observing that both $N_{X}$ and $\mathbb{S}$ are associated to the Spin principal bundle, $\tilde{P}$, of $X$, which is the double cover of the frame bundle of $X$, with the same representation. The proof of this is similar to that for standard calibrations and it has been described in $[19,29]$. Here we shall summarize the proof. First observe that every oriented three manifold admits a spin structure and so $\tilde{P}$ exists for all associative calibrations $X$. Then observe that the seven-dimensional representation $\rho_{7}$ of $G_{2}$ which leaves the three-form $\psi$ invariant decomposes as $\rho_{7}=v_{3} \oplus s_{4}$, where $v_{3}$ is the three-dimensional representation of $S U(2)$ induced by the standard threedimensional vector representation of $S O(3)$ acting on the directions 123 and $s_{4}$ is the four-dimensional real spinor representation of $S U(2)$ acting on the directions 4567 . Since 4567 are the normal directions of $X$, the normal bundle $N_{X}$ and the spin bundle $\mathbb{S}$ are associated to $\tilde{P}$ with the same representation $s_{4}$, so $N_{X}=\mathbb{S}$. Therefore the deformation equation (10.109) is the Dirac equation in three-dimensions with respect to the connection $\tilde{\nabla}$; the gamma-matrices are given by the $\left\{\Omega_{i} ; i=1,2,3\right\}$. This is in fact an elliptic differential equation and so if a moduli exist, the moduli space is finite dimensional.

The index of the Dirac operator that appears in the deformations of generalized associative calibrations vanishes. Because of this it is expected that generic generalized associative calibrations will not have moduli. This is similar to the case of standard associative calibrations. Although generic generalized associative calibrations do not have moduli, we shall find many examples of families of generalized associative calibrations in special cases. 
11. Special Cases. There are several special cases of $G_{2}$ structures according to Fernandez-Gray classification [9] depending on various additional conditions that the three-form $\psi$ and its dual $* \psi$ satisfy.

Calibrated and cocalibrated $G_{2}$ manifolds

The manifold $(M, g, \psi)$ is calibrated if $d \psi=0$ and $(M, g, \psi)$ is cocalibrated if $d * \psi=0$. It is known that if $(M, g, \psi)$ is both calibrated and cocalibrated, then the holonomy of the Levi-Civita connection $\nabla^{g}$ is contained in $G_{2}$.

For calibrated $G_{2}$ manifolds, the generalized associative submanifolds are minimal because $\psi$ is closed. In addition, the deformation equations of such submanifolds are given by the Dirac equation in (10.109) with respect to a connection of the normal bundle which is induced from a connection on $M$ which has non-vanishing torsion.

For calibrated $G_{2}$ manifolds, the generalized co-associative submanifolds generically are not minimal because $* \psi$ is not closed. The deformations of such submanifolds are given in (9.101) or in (9.103). Because $d \psi=0$, the latter equation can be simplified to

$$
d \alpha_{V}=0 .
$$

Since $\alpha_{V}$ is anti-self-dual, the dimension of the moduli space of generalized coassociative calibrations in calibrated $G_{2}$ manifolds is $b_{2}^{-}$.

For cocalibrated $G_{2}$ manifolds, the generalized associative submanifolds are not generically minimal because $\psi$ is not closed. The deformation equations of such submanifolds are given by the Dirac equation (10.109) with respect to a connection of the normal bundle which is induced from a connection on $M$, which has non-vanishing torsion.

For cocalibrated $G_{2}$ manifolds, the generalized co-associative submanifolds are minimal because $d * \psi=0$. The deformations of such submanifolds are given in (9.101) or in (9.103).

Another type of $G_{2}$ manifold for which the associated generalized calibrations can be analyzed as for co-calibrated $G_{2}$ manifolds is that of cocalibrated $G_{2}$ manifolds of pure type. For such manifolds $d * \psi=0$ and $d \psi \wedge \psi=0$. Again the co-associative calibrations are minimal.

Integrable $G_{2}$ manifolds

The manifold $(M, g, \psi)$ is an integrable $G_{2}$ manifold iff

$$
d * \psi=\theta \wedge * \psi
$$

where $3 \theta=-*(* d \psi \wedge \psi)$ is the Lee form. It has been shown in [10] that such $G_{2}$ manifolds admit a unique connection with torsion a three-form.

For generic integrable $G_{2}$ manifolds, both $\psi$ and $* \psi$ are not closed, so the generalized associative and co-associative calibrations are not minimal. The deformation equations for generalized associative calibrations are given by the Dirac equation (10.109) but in this case the connection on the normal bundle is induced from a connection with torsion a three-form on $M$. The deformation equations for generalized co-associative calibrations (9.103) can be simplified somewhat using the expression for the torsion in [10]. In particular, denoting the torsion 3 -form by $T=H$, we have [10] that

$$
d \psi=\frac{1}{6}(d \psi . * \psi) * \psi+\theta \wedge \psi+* H
$$


Hence, noting that $\left.i_{V} * \psi\right|_{X}=0$, it follows that

$$
\left.i_{V} d \psi\right|_{X}=\left.\left(-\theta \wedge \alpha_{V}+i_{V}(* H)\right)\right|_{X}
$$

and so we require that

$$
d \alpha_{V}-\theta \wedge \alpha_{V}+\frac{1}{36}\left(\alpha_{V}\right)_{a_{1} a_{2}} \psi^{i a_{1} a_{2}} * H_{i a b c} e^{a} \wedge e^{b} \wedge e^{c}=0
$$

However, despite this simplification, it has not been possible to compute the dimension of the moduli space.

The subclass of integrable $G_{2}$ manifolds which have applications in physics (string theory) are those for which the 1 -form $\theta$ is exact and so $\theta=-2 d \Phi$ for some function $\Phi$ on $M$ which is identified with the dilaton. The analysis of generalized associative and co-associative calibrations in this case is as for the integrable $G_{2}$ manifolds above. There is some additional simplification though in the deformation equations for generalized co-associative calibrations. In particular, defining $\rho_{V}=e^{2 \Phi} \alpha_{V}$ and substituting in (11.114), we obtain

$$
d \rho_{V}+\frac{1}{36}\left(\rho_{V}\right)_{a_{1} a_{2}} \psi^{i a_{1} a_{2}} * H_{i a b c} e^{a} \wedge e^{b} \wedge e^{c}=0
$$

Nearly parallel or weak holonomy $G_{2}$ manifolds

The manifold $(M, g, \psi)$ admits a nearly parallel or weak holonomy $G_{2}$ structure iff $d \psi=\lambda * \psi$, for $\lambda$ constant. If $\lambda=0$, then $(M, g, \psi)$ is calibrated. If $\lambda \neq 0$, then $(M, g, \psi)$ is co-calibrated. Since we have already investigated the case of calibrated $G_{2}$ manifolds, we shall focus on the case that $\lambda \neq 0$. In [10], it has been shown that nearly parallel manifolds admit a connection $\nabla$ with torsion a three-form. In particular $T=-\frac{1}{6} \lambda \psi$.

There are many examples of nearly parallel $G_{2}$ manifolds which include $S^{7}$, $S O(5) / S O(3)$ and the Aloff-Wallach spaces $N(n, m)=S U(3) / U(1)_{n, m}$; the embedding of $U(1)$ in $S U(3)$ will be described later.

For nearly parallel $G_{2}$ manifolds, the generalized associative submanifolds are not generically minimal because $\psi$ in not closed. The deformation equations of such submanifolds are given by the Dirac equation (10.109). Using the connection with the torsion the three-form which is proportional to $\psi$, the deformation equation can be simplified to

$$
\sum_{i, b}\left(J_{i}\right)_{b}^{a} \nabla_{i}^{g} V^{b}+\frac{\lambda}{4} V^{a}=0 .
$$

Therefore the deformations of the associative submanifolds are eigenspinors of the Dirac operator.

There are no compact without boundary generalized co-associative submanifolds in nearly parallel $G_{2}$ manifolds. Although in this case the calibration form $* \psi$ is closed, it is also exact and therefore the volume of such a calibrated submanifold is zero $^{1}$.

\footnotetext{
${ }^{1}$ We thank D. Joyce for pointing this out to us.
} 


\section{Examples.}

12.1. A group manifold example. Consider the group manifold $M=S^{3} \times$ $\tilde{S}^{3} \times S^{1}$ with left-invariant metric

$$
g=\sum_{i}\left(\sigma^{i}\right)^{2}+\sum_{i}\left(\tilde{\sigma}^{i}\right)^{2}+\left(\sigma^{0}\right)^{2}
$$

and equipped with the left-invariant three-form

$$
\psi=\sigma^{123}+\sigma^{1} \wedge\left(\tilde{\sigma}^{01}-\tilde{\sigma}^{23}\right)+\sigma^{2} \wedge\left(\tilde{\sigma}^{02}+\tilde{\sigma}^{13}\right)+\sigma^{3} \wedge\left(\tilde{\sigma}^{03}-\tilde{\sigma}^{12}\right),
$$

where $\left\{\sigma^{i} ; 1,2,3\right\}$ and $\left\{\tilde{\sigma}^{i} ; 1,2,3\right\}$ are the left-invariant one-forms on the three-spheres $S^{3}$ and $\tilde{S}^{3}$ in $M$, respectively and $\tilde{\sigma}^{0}$ is the invariant one-form on $S^{1}$. Clearly this three-form $\psi$ defines a $G_{2}$ structure on $M$ which is parallel with respect to the $\nabla$ connection on the group manifold associated with the left action.

It can be easily seen that the submanifold $S^{3}$ is a generalized associative calibration, while $S^{1} \times \tilde{S}^{3}$ is a generalized co-associative calibration.

Observe that the submanifolds $S^{3} \times\{p\}, p \in \tilde{S}^{3} \times S^{1}$ are all generalized associative calibrations and so the moduli space has dimension at least four. In fact the moduli space has dimension exactly four. To see this observe that the equation for the deformations in this case is

$$
\sum_{i, b}\left(\Omega_{i}\right)_{a b} \tilde{\nabla}_{i} V^{b}=\sum_{i, b}\left(\Omega_{i}\right)_{a b} \nabla_{i} V^{b}=0
$$

and $\nabla$ is a flat connection. Therefore

$$
\nabla^{2} V^{a}=0
$$

Then

$$
\int_{S^{3}}(\nabla V, \nabla V)=-\int_{S^{3}}\left(V, \nabla^{2} V\right)=0
$$

and hence $V$ is constant. So the moduli space has dimension four. In fact the moduli space in this case is $\tilde{S}^{3} \times S^{1}$ and therefore $M$ is a family of generalized associative calibrations.

Similarly, observe that the submanifolds $\tilde{S}^{3} \times S^{1} \times\{p\}, p \in S^{3}$, are all generalized co-associative calibrations and so the moduli space in this case has dimension at least three. In fact the moduli space has dimension exactly three. The deformation equation is

$$
\sum_{i, b}\left(J_{i}\right)^{b}{ }_{a} \tilde{\nabla}_{b} V^{i}=\sum_{i, b}\left(J_{i}\right)_{a}^{b} \nabla_{b} V^{i}=\sum_{b} \nabla^{b}\left(\alpha_{V}\right)_{b a}=0 .
$$

Therefore $\alpha_{V}$ is co-closed with respect to the flat connection $\nabla$. Since $\alpha_{V}$ is antiself-dual, it is also closed, $\nabla \wedge \alpha_{V}=0$, with respect to $\nabla$. Since $\alpha_{V}$ is both closed and co-closed is harmonic with respect to the Laplacian $\nabla^{2}$. A partial integration argument similar to the one above implies that $\alpha_{V}$ is necessarily $\nabla$-parallel. This implies that the dimension of the moduli space is three. In fact in this case, the moduli space is $S^{3}$. 
Generalized calibrations in $S^{7}=S p(2) / S p(1)$

12.2. Generalized associative calibrations in homogeneous spaces. Identify $\mathbb{R}^{8}=\mathbb{H}^{2}$. Then observe that the action of $S p(2)$ preserves the equation for $S^{7}$ written in terms of quaternions with stability subgroup $S p(1)$ up to a conjugation. This implies that $S p(2) / S p(1)=S^{7}$. In addition observe that $S p(1) \subset$ $S p(1) \times S p(1) \subset S p(2)$. This leads to the principal fibration $S p(1) \rightarrow S^{7} \rightarrow \mathbb{H} P^{1}$. This is the principal fibration associated with the anti-self dual $S U(2)=S p(1)$ instanton connection in $S^{4}=\mathbb{H} P^{1}$. Let $\left\{\alpha^{i} ; i=1,2,3\right\}$ be the associated connection with curvature

$$
\omega^{i}=d \alpha^{i}+\epsilon_{j k}^{i} \alpha^{j} \wedge \alpha^{k}
$$

The Bianchi identity implies that

$$
d \omega^{i}=2 \epsilon^{i}{ }_{j k} \omega^{j} \wedge \alpha^{k}
$$

In addition there is a local frame $\left\{\ell^{a} ; a=4, \ldots, 7\right\}$ such that

$$
\omega^{i}=\frac{1}{2} \Omega_{a b}^{i} \ell^{a} \wedge \ell^{b}
$$

where $\left\{\Omega^{i} ; i=1,2,3\right\}$ is the basis of constant anti-self-dual two-forms in $\mathbb{R}^{4}$ given in (9.99) and (9.100).

Next consider the metric and the three-form on $S^{7}$

$$
\begin{aligned}
d s^{2} & =y^{2} \sum_{i=1}^{3}\left(\alpha^{i}\right)^{2}+z^{2} \sum_{a=4}^{7}\left(\ell^{a}\right)^{2} \\
\psi & =y^{3} \alpha^{1} \wedge \alpha^{2} \wedge \alpha^{3}+y z^{2} \sum_{i=1}^{3} \alpha^{i} \wedge \omega^{i},
\end{aligned}
$$

where $y, z \in \mathbb{R}-\{0\}$. It can be easily seen by setting $\left\{e^{i}=y \alpha^{i} ; i=1,2,3\right\}$ and $\left\{e^{i}=z \ell^{i} ; i=4, \ldots, 7\right\}$ that the metric $d s^{2}$ and $\psi$ above take the canonical form of a $G_{2}$ structure as in (9.99) and (9.100).

The fibres of the fibration $S p(1) \rightarrow S^{7} \rightarrow \mathbb{H} P^{1}$ are all associative generalized calibrations; this can easily be seen by observing that

$$
\left.\psi\right|_{S p(1)}=\left.y^{3}\left(\alpha^{1} \wedge \alpha^{2} \wedge \alpha^{3}\right)\right|_{S p(1)}=d \operatorname{vol}(S p(1)) .
$$

This is the case for any $y, z \in \mathbb{R}-\{0\}$. Therefore this fibration is a family of generalized associative calibrations.

It can be easily seen that the $G_{2}$ structure on $S^{7}$ in (12.126) is nearly parallel, $d \psi=\lambda * \psi$, iff

$$
\begin{aligned}
-3 y & =\lambda z^{2} \\
\frac{1}{2} y^{2}+z^{2} & =-\frac{1}{2} \lambda y z^{2} .
\end{aligned}
$$

This system has a solution for $y=-3 / \lambda$ and $z= \pm 3 / \lambda$. This gives a nearly parallel $G_{2}$ manifold which is the squashed $S^{7}$. Clearly the squashed $S^{7}$ is a family of generalized associative calibrations. 
REMARK 3. The Hopf fibration $S^{1} \rightarrow S^{3} \rightarrow S^{2}$ is also a smooth family of generalized calibrations. To see this observe that the metric on $S^{3}$ can be written as

$$
d s^{2}\left(S^{3}\right)=\left(\sigma^{3}\right)^{2}+\left(\sigma^{1}\right)^{2}+\left(\sigma^{2}\right)^{2}
$$

where $\sigma^{1}, \sigma^{2}, \sigma^{3}$ are the left-invariant one-forms on $S^{3}$ and $d s^{2}\left(S^{2}\right)=\left(\sigma^{1}\right)^{2}+\left(\sigma^{2}\right)^{2}$. It can be easily seen that $\sigma^{3}$ is a generalized calibration in $S^{3}$ of degree one. The calibrated lines are circles which are the fibres of the Hopf-fibration. Therefore $S^{3}$ is a family of generalized degree one calibrations with space of parameters $S^{2}$.

Generalized calibrations in $M=S O(5) / S O(3)$

We shall demonstrate that $M=S O(5) / S O(3)$ is a family of generalized associative calibrations. We remark that $M$ is not homeomorphic to $S^{7} ; M$ and $S^{7}$ have the same deRham cohomology but $M$ exhibits torsion in the third cohomology. Observe that $s o(5)=s o(4) \oplus \mathbb{R}^{4}$ and $s o(4)$ acts with the fundamental representation on $\mathbb{R}^{4}$. Since $s o(4)=s o(3) \oplus s o(3)$, the structure constants decompose under the decomposition $\Lambda \mathbb{R}^{4}=\Lambda^{2+} \mathbb{R}^{4} \oplus \Lambda^{2-} \mathbb{R}^{4}$. Under this decomposition of $s o(5)$ a frame can be introduced at $M$ which satisfies the following structure equations:

$$
\begin{aligned}
d e^{a} & =\left(J_{i}\right)^{a}{ }_{b} \rho^{i} \wedge e^{b}+\left(I_{i}\right)^{a}{ }_{b} \sigma^{i} \wedge e^{b} \\
d \rho^{i} & =\epsilon_{j k}^{i} \rho^{i} \wedge \rho^{j}-\frac{1}{2}\left(\Omega_{i}\right)_{a b} e^{a} \wedge e^{b},
\end{aligned}
$$

where $\left\{e^{a} ; a=1, \ldots, 4\right\}$ are associated with a basis in $\mathbb{R}^{4},\left\{\sigma^{i} ; i=1,2,3\right\}$ are associated with a basis in the Lie algebra of the stability subgroup of the coset and $\left\{\rho^{i} ; i=1,2,3\right\}$ are the rest of the generators. The structure constant matrices $\left\{J_{i} ; i=1,2,3\right\}$ are anti-self-dual and the structure constants $\left\{I_{i} ; i=1,2,3\right\}$ are self-dual; $\left(\Omega_{i}\right)_{a b}=\delta_{a c}\left(J_{i}\right)^{c}$.

The metric and three-form on $M$ are the following:

$$
\begin{aligned}
d s^{2}\left(S^{7}\right) & =\sum_{i=1}^{3}\left(\rho^{i}\right)^{2}+\sum_{a=1}^{4}\left(e^{a}\right)^{2} \\
\psi & =\rho^{1} \wedge \rho^{2} \wedge \rho^{3}+2 \sum_{i=1}^{3}\left(\Omega_{i}\right)_{a b} \rho^{i} \wedge e^{a} \wedge e^{b} .
\end{aligned}
$$

Observe that both are invariant under the stability group $S O(3)$ of the coset and so they are globally defined on $M$. It can be easily seen that the data given in (12.133) define a nearly parallel $G_{2}$ structure on $M$.

Next consider the obvious subgroups of $S O(5) ; S O(3) \subset S O(4) \subset S O(5)$. Then there is a fibration $S O(4) / S O(3) \rightarrow S O(5) / S O(3) \rightarrow S O(5) / S O(4)$ or equivalently $S^{3} \rightarrow M \rightarrow S^{4}$. The cotangent bundles of the fibres at every point $p \in S^{4}$ are spanned by $\left\{\rho^{i} \mid p ; i=1,2,3\right\}$ and because the metric on $M$ is diagonal in this basis all the $S^{3}$ fibres of this fibration are associative submanifolds of $M$. This fibration is a family of generalized associative $G_{2}$ calibrations.

Generalized calibrations in Aloff-Wallach spaces $N(n, m)$

Another class of nearly parallel $G_{2}$ manifolds are the so called Aloff-Wallach spaces $N(n, m)=S U(3) / U(1)_{n, m}$. The $U(1)$ is embedded in $S U(3)$ as

$$
\operatorname{diag}\left(e^{i n \chi}, e^{i m \chi}, e^{-i(n+m) \chi}\right),
$$


where $n, m \in \mathbb{Z}$. To construct the $G_{2}$ structures on this space write $s u(3)=u(1) \oplus \mathbb{R}^{7}$. Under the action of $U(1), \mathbb{R}^{7}$ decomposes as $\mathbb{R}^{7}=\mathbb{R}^{2} \oplus \mathbb{R}^{2} \oplus \mathbb{R}^{2} \oplus \mathbb{R}$. This can be seen by using the action of $u(1)$ on the Cartan subalgebra and the step operators of $s u(3)$. In particular, each $\mathbb{R}^{2}$ is spanned by the step operators $E_{ \pm \alpha}$, where $\alpha$ is a positive root, while $\mathbb{R}$ is spanned by the direction in the Cartan subalgebra of $s u(3)$ which is orthogonal to the generator of the embedded $u(1)$. A local frame can be introduced on $N(n, m)$ according to this decomposition as $\left\{\sigma^{i}, \rho^{i}, \zeta^{i}, \eta ; i=1,2\right\}$. To be precise, let $\left\{L_{B}{ }^{A} ; A, B=1,2,3\right\},\left(L_{B}{ }^{A}\right)^{\dagger}=L_{B}{ }^{A}, \operatorname{tr} L_{A}{ }^{A}=0$, be the left invariant forms on $S U(3), d L_{A}{ }^{B}=i L_{a}{ }^{C} \wedge L_{C}{ }^{A}$. We set $\sigma^{1}+i \sigma^{2}=i L_{3}{ }^{1}, \rho^{1}+i \rho^{2}=L_{2}{ }^{3} \zeta^{1}+i \zeta^{2}=L_{1}{ }^{2}$, and $\eta=\sqrt{2}\left(\cos \delta L_{1}{ }^{1}+\sin \delta L_{2}{ }^{2}\right)$, where $\tan \delta=-\frac{n}{m}$. This decomposition is similar to that in [7]. A metric on $N(n, m)$ can be written as

$$
d s^{2}=x^{2} \sum_{i}\left(\sigma^{i}\right)^{2}+y^{2} \sum_{i}\left(\rho^{i}\right)^{2}+z^{2} \sum_{i}\left(\zeta^{i}\right)^{2}+f^{2} \eta^{2}
$$

where $x, y, x, f \in \mathbb{R}-\{0\}$. To define a $G_{2}$ three-form, it is most convenient to induce it from a Kähler form $\omega$ and a $(3,0)$-form $\phi$ on $\mathbb{R}^{6}$ because of the above decomposition of $\mathbb{R}^{7}$. Indeed consider the two-form

$$
\omega=x^{2} \sigma^{1} \wedge \sigma^{2}+y^{2} \rho^{1} \wedge \rho^{2}+z^{2} \zeta^{1} \wedge \zeta^{2}
$$

and the $(3,0)$-form

$$
\phi=x y z\left(\sigma^{1}+i \sigma^{2}\right) \wedge\left(\rho^{1}+i \rho^{2}\right) \wedge\left(\zeta^{1}+i \zeta^{2}\right) .
$$

Then the $G_{2}$ three-form on $N(n, m)$ can be defined as

$$
\psi=\operatorname{Re} \phi-f \eta \wedge \omega .
$$

Setting $e^{1}=x \sigma^{1}, e^{5}=x \sigma^{2}, e^{2}=y \rho^{1}, e^{6}=y \rho^{2}, e^{3}=z \zeta^{1}, e^{7}=z \zeta^{2}, e^{4}=f \eta$, one can bring the metric $d s^{2}$ and $\psi$ above into the canonical form of a $G_{2}$ structure given in (9.99) and (9.100).

For all $x, y, z, f \in \mathbb{R}-\{0\}$, the above data define a $G_{2}$ structure on $N(n, m)$. However not all these $G_{2}$ structures are nearly parallel. It can be shown that if

$$
\begin{aligned}
\lambda & =\left(x^{2}+y^{2}+z^{2}\right) \\
4 x y z+2 \sqrt{2} f\left(y^{2}(\cos \delta-\sin \delta)+z^{2} \sin \delta\right) & =\lambda y^{2} z^{2} \\
4 x y z+2 \sqrt{2} f\left(x^{2}(\cos \delta-\sin \delta)-z^{2} \cos \delta\right) & =\lambda x^{2} z^{2} \\
4 x y z+2 \sqrt{2} f\left(x^{2} \sin \delta-y^{2} \cos \delta\right) & =\lambda y^{2} x^{2}
\end{aligned}
$$

then the $G_{2}$ structure is nearly parallel. It is known that these equations have solutions and so there are nearly parallel $G_{2}$ structures on $N(n, m)$; for a recent discussion see $[6,7]$.

To find generalized $G_{2}$ calibrated submanifolds in $N(n, m)$, observe that $U(1)_{n, m} \subset S(U(2) \times U(1)) \subset S U(3)$. Viewing $S(U(2) \times U(1))$ as a $3 \times 3$ matrix, the embedding of $U(1)$ in $S(U(2) \times U(1))$ is as in (12.134). This sequence of subgroups of $S U(3)$ define the fibration

$$
S(U(2) \times U(1)) / U(1)_{n, m} \rightarrow N(n, m) \rightarrow \mathbb{C} P^{2} .
$$

In fact it turns out that the typical fibre is $S(U(2) \times U(1)) / U(1)_{n, m}=S^{3} / \mathbb{Z}_{p}$, where $p=|n+m|>0$; for $p=0$ the typical fibre is $S^{2} \times S^{1}$. 
Next decompose $s(u(2) \oplus u(1))$ under the action of $u(1)$ as $s(u(2) \oplus u(1))=$ $u(1) \oplus \mathbb{R}^{3}$. Moreover $\mathbb{R}^{3}$ decomposes under the irreducible two dimensional real representation of $u(1)$ as $\mathbb{R}^{3}=\mathbb{R}^{2} \oplus \mathbb{R}$. Since $s(u(2) \oplus u(1)) \subset s u(3), \mathbb{R}^{3}$ is a submodule of $\mathbb{R}^{7}$ under the action of $u(1)$. Therefore it can be arranged such that the tangent space of the fibres of the fibration $S^{3} / \mathbb{Z}_{p} \rightarrow N(n, m) \rightarrow \mathbb{C} P^{2}$ is spanned by $\left\{\eta, \sigma^{1}, \sigma^{2}\right\}$. It is clear that $\left.\psi\right|_{S^{3} / \mathbb{Z}_{p}}=d \operatorname{vol}\left(S^{3} / \mathbb{Z}_{p}\right)$ and so every fibre is a generalized associative calibration. Therefore the Aloff-Wallach spaces are families of generalized associative calibrations for any $G_{2}$ structure defined in (12.135) and (12.138).

13. Deformations of generalized Cayley Calibrations. Let $(M, g)$ be an eight-dimensional Riemannian manifold which admits a metric connection $\nabla$ with holonomy contained in $\operatorname{Spin}(7)$. On such a manifold there is a local frame $\left\{e^{A} ; A=\right.$ $1, \ldots, 8\}$ such that the self-dual four-form

$\Phi=e^{1234}+\left(e^{12}-e^{34}\right) \wedge\left(e^{56}-e^{78}\right)+\left(e^{13}+e^{24}\right) \wedge\left(e^{57}+e^{68}\right)+\left(e^{14}-e^{23}\right) \wedge\left(e^{58}-e^{67}\right)+e^{5678}$

is $\nabla$-parallel.

The condition that a four-dimensional submanifold $X \subset M$ is calibrated with respect to $\Phi$ is that $\left.\tau\right|_{X}=0$ where $\tau \in \Omega^{4}(M, F)$ is a four-form which takes values on the vector bundle $F ; F=P \times_{d_{7}} \mathbb{R}^{7}$ where $d_{7}$ is the seven-dimensional representation of $\operatorname{Spin}(7)$, i.e. the one induced from the standard seven-dimensional vector representation of $S O(7)$. This four-form $\tau$ is associated with the four-fold cross product of $\mathbb{R}^{8}=\mathbb{O}$ with values in $\operatorname{Im} \mathbb{O}$ and it is $\operatorname{Spin}(7)$ invariant, so $\tau$ is $\nabla$-parallel Explicitly, in an appropriate basis, $\tau$ is

$$
\tau=\left(\begin{array}{c}
\left(e^{14}-e^{23}\right) \wedge\left(e^{57}+e^{68}\right)-\left(e^{13}+e^{24}\right) \wedge\left(e^{58}-e^{67}\right) \\
\left(e^{12}-e^{34}\right) \wedge\left(e^{58}-e^{67}\right)-\left(e^{14}-e^{23}\right) \wedge\left(e^{56}-e^{78}\right) \\
\left(e^{13}+e^{24}\right) \wedge\left(e^{56}-e^{78}\right)-\left(e^{12}-e^{34}\right) \wedge\left(e^{57}+e^{68}\right) \\
e^{2345}-e^{1346}+e^{1247}-e^{1238}+e^{1678}-e^{2578}+e^{3568}-e^{4567} \\
e^{2346}+e^{1345}+e^{1248}+e^{1237}-e^{2678}-e^{1578}-e^{4568}-e^{3567} \\
e^{2347}+e^{1348}-e^{1245}-e^{1236}-e^{3678}-e^{4578}+e^{1568}+e^{2567} \\
e^{2348}-e^{1347}-e^{1246}+e^{1235}-e^{4678}+e^{3578}+e^{2568}-e^{1567}
\end{array}\right)
$$

The Cayley calibration is a degree four calibration in an eight-manifold $M$. Let $X$ be a generalized Cayley submanifold whose tangent directions are spanned by $\left\{e^{a} ; a=1, \ldots, 4\right\}$ and normal directions by $\left\{e^{i} ; i=5, \ldots, 8\right\}$. Then, by the same reasoning used for the generalized associative deformations, the condition $\left.\mathcal{L}_{V} \tau\right|_{X}=0$ implies that

$$
\mathcal{D} V_{i}:=\sum_{a, j} t_{i j}^{a} \tilde{\nabla}_{a} V^{j}=0
$$

where $\left\{t^{a} ; a=1, \ldots 4\right\}=\left\{1, \Omega_{r} ; r=1,2,3\right\}$, and $\left\{\Omega_{r} ; r=1,2,3\right\}$ is a basis of constant anti-self-dual 2-forms in $\mathbb{R}^{4}$ spanned by the directions $5,6,7,8$; such a basis has been defined in section nine for the $G_{2}$ calibrations. The operator $\mathcal{D}$ is elliptic. So if the moduli space exists, it is finite dimensional. It is expected that for generic generalized Cayley cycles, the dimension of the moduli space is the index of the operator $\mathcal{D}$. The index of this operator $\mathcal{D}$ is the same as that computed for the standard Cayley calibrations because the principal symbol is the same. It has been found [25] that $\operatorname{ind}(\mathcal{D})=\sigma(X)-\frac{1}{2} \chi(X)-\frac{1}{2}[X] \cdot[X]$, where $\sigma(X), \chi(X)$ and $([X] \cdot[X])$ is the signature, Euler number and self-intersection of the Cayley calibration $X$. 


\section{Examples.}

14.1. A group manifold example. Let $M=S^{3} \times \tilde{S}^{3} \times S^{1} \times \tilde{S}^{1}$ equipped with the left invariant metric

$$
g=\sum_{i=1}^{3}\left(\sigma^{i}\right)^{2}+\sum_{i=1}^{3}\left(\tilde{\sigma}^{i}\right)^{2}+\left(\sigma^{0}\right)^{2}+\left(\tilde{\sigma}^{0}\right)^{2}
$$

and the left invariant self-dual four-form

$$
\begin{aligned}
\Phi= & \sigma^{0123}+\left(\sigma^{01}-\sigma^{23}\right) \wedge\left(\tilde{\sigma}^{01}-\tilde{\sigma}^{23}\right)+\left(\sigma^{02}\right. \\
& \left.+\sigma^{13}\right) \wedge\left(\tilde{\sigma}^{02}+\tilde{\sigma}^{13}\right)+\left(\sigma^{03}-\sigma^{12}\right) \wedge\left(\tilde{\sigma}^{03}-\tilde{\sigma}^{12}\right)+\tilde{\sigma}^{0123}
\end{aligned}
$$

where $\left\{\sigma^{i} ; i=1,2,3\right\}$ and $\left\{\tilde{\sigma}^{i} ; i=1,2,3\right\}$ are the left-invariant one-forms of $S^{3}$ and $\tilde{S}^{3}$, respectively, and $\sigma^{0}$ and $\tilde{\sigma}^{0}$ is the bi-invariant one-form of $S^{1}$ and $\tilde{S}^{1}$, respectively. Both the metric and self-dual four-form are parallel with respect to the connection associated with the left-action. With an appropriate choice of orientation of $M$ both submanifolds $S^{1} \times S^{3}$ and $\tilde{S}^{1} \times \tilde{S}^{3}$ are generalized Cayley calibrations. We shall focus on the investigation of the moduli space of the $S^{1} \times S^{3}$ calibration; the study of the moduli space of $\tilde{S}^{1} \times \tilde{S}^{3}$ is similar. Observe that all the submanifolds $S^{1} \times S^{3} \times\{p\}$, where $p \in \tilde{S}^{1} \times \tilde{S}^{3}$ are Cayley calibrations. Therefore the dimension of the moduli space is at least four. In fact the dimension of the moduli space is exactly four. To see this one uses the fact that $\tilde{\nabla}=\partial$ acting on the normal vector fields of the calibration. Then the result follows from a partial integration argument as in the group manifold example in the $G_{2}$ case. The moduli space of the $S^{1} \times S^{3}$ Cayley calibration is $\tilde{S}^{1} \times \tilde{S}^{3}$. So $M$ is a family of generalized calibrations for which the fibers and the base are calibrated.

This example is a special case of a larger class of examples which can be constructed by taking a seven-dimensional manifold $(N, g, \psi)$ with a $G_{2}$ structure which admits a associative submanifold $X$. Then the manifold $M=N \times S^{1}$ with $d s^{2}(M)=d s^{2}\left(S^{1}\right)+d s^{2}(N)$ and $\Phi=e^{0} \wedge \psi+* \psi$ is a $\operatorname{Spin}(7)$ manifold; $*$ is the Hodge operation in $N$ and $e^{0}$ is the invariant one-form along $S^{1}$. In addition $S^{1} \times X$ is a generalized Cayley calibration.

15. The $\partial \bar{\partial}$-lemma and some useful formulae. The main tool that we shall use for the investigation of hermitian manifolds with trivial canonical bundle is the $\partial \bar{\partial}$-lemma. This can be stated as follows. Let $(M, g, J)$ be a hermitian manifold, and $\phi$ and $\tilde{\phi}$ be two closed (p,q)-forms. Locally one can always write

$$
\phi=\tilde{\phi}+\partial \bar{\partial} \psi
$$

for some locally defined ( $\mathrm{p}-1, \mathrm{q}-1)$-form $\psi$. The $\partial \bar{\partial}$-lemma states that if $\phi$ and $\tilde{\phi}$ represent the same class in the Dolbeault cohomology, then (15.146) is valid for some (p-1, q-1)-form on $M$.

Let $(M, g, J)$ be a $2 n$-dimensional $(n>1)$ Hermitian manifold with complex structure $J$ and compatible Riemannian metric $g$. Denote the Kähler form by $\Omega$. The definitions $J X$ and $J \alpha$, for $X$ a vector field and $\alpha$ a one-form, are $(J X)^{i}=$ $J^{i}{ }_{j} X^{j}, \quad(J \alpha)^{i}=-(\alpha \circ J)^{i}=-\alpha_{k} J^{k i}$ respectively. The Lee form $\theta$ is defined by

$$
\theta=d^{\dagger} \Omega \circ J \quad \theta_{i}=-\left(\nabla^{g}\right)^{k} \Omega_{k j} J^{j}{ }_{i}
$$

where $d^{\dagger}$ is the adjoint of $d$ and $\nabla^{g}$ is the Levi-Civita connection of the metric $g$. Equivalently $d^{\dagger} \Omega=J \theta$. If the Lee form $\theta=0$ then the hermitian manifold is said to be balanced. Balanced hermitian manifolds are studied in $[30,13,1,2,12,11]$. 
The Bismut connection $\nabla^{b}$ and the Chern connection $\nabla^{c}$ are given by

$$
\begin{aligned}
& g\left(\nabla_{X}^{b} Y, Z\right)=g\left(\nabla_{X}^{g} Y, Z\right)+\frac{1}{2} d^{c} \Omega(X, Y, Z), \\
& g\left(\nabla_{X}^{c} Y, Z\right)=g\left(\nabla_{X}^{g} Y, Z\right)+\frac{1}{2} d \Omega(J X, Y, Z),
\end{aligned}
$$

respectively. Recall that $d^{c}=i(\bar{\partial}-\partial)$. In particular, $d^{c} \Omega(X, Y, Z)=$ $-d \Omega(J X, J Y, J Z)$.

Let $\rho^{b}$ and $\rho^{c}$ be the Ricci forms of the Bismut and Chern connections respectively. Then it was shown in [3] that

$$
\rho^{c}=\rho^{b}+d(J \theta)
$$

In complex coordinates $\left\{z^{\alpha}\right\}, \alpha=1, \ldots, n$, we have the following formulae:

Let $\stackrel{c}{\Gamma}$ be the Chern connection. Then

$$
(\stackrel{c}{\Gamma})_{\alpha \beta}^{\delta}=g^{\bar{\gamma} \delta} \partial_{\alpha} g_{\beta \bar{\gamma}}
$$

and so

$$
(\stackrel{c}{\Gamma})_{\alpha}=(\stackrel{c}{\Gamma})_{\alpha \beta}^{\beta}=\partial_{\alpha}(\log (\operatorname{det}(g))
$$

The Lee form is then

$$
\theta_{\alpha}=(\stackrel{c}{\Gamma})_{\alpha \beta}^{\beta}-(\stackrel{c}{\Gamma})_{\beta \alpha}^{\beta}=g^{\beta \bar{\gamma}}\left(\partial_{\alpha} g_{\beta \bar{\gamma}}-\partial_{\beta} g_{\alpha \bar{\gamma}}\right)=\partial_{\alpha}\left(\log (\operatorname{det}(g))-g^{\beta \bar{\gamma}} \partial_{\beta} g_{\alpha \bar{\gamma}}\right.
$$

In terms of the Chern connection, the Lee form is

$$
(d \theta)_{\alpha \gamma}=\partial_{\alpha}(\stackrel{c}{\Gamma})_{\beta \gamma}^{\beta}-\partial_{\gamma}(\stackrel{c}{\Gamma})_{\beta \alpha}^{\beta}
$$

The Ricci form of the Chern connection is $\rho^{c}$

$$
i \rho_{\bar{\beta} \alpha}^{c}=\partial_{\bar{\beta}} D_{\alpha \sigma}^{\sigma}=\partial_{\bar{\beta}} \partial_{\alpha}\left(\log (\operatorname{det}(g))=\partial_{\bar{\beta}}\left(g^{\bar{\gamma} \delta} \partial_{\alpha} g_{\delta \bar{\gamma}}\right)\right.
$$

The (1,1)-part of formula (15.150) can be written in the following way [22]

$$
i \rho_{\bar{\beta} \alpha}^{b}=i \rho_{\bar{\beta} \alpha}^{c}-\left(\partial_{\bar{\beta}} \theta_{\alpha}+\partial_{\alpha} \theta_{\bar{\beta}}\right)
$$

Using (15.153), (15.155) we obtain that (15.150) is equivalent to the following two formulae

$$
\begin{gathered}
i \rho_{\bar{\beta} \alpha}^{b}=\partial_{\bar{\beta}}\left(g^{\sigma \bar{\gamma}} \partial_{\sigma} g_{\alpha \bar{\gamma}}\right)-\partial_{\alpha}\left(g^{\sigma \bar{\gamma}} \partial_{\bar{\beta}} g_{\sigma \bar{\gamma}}\right)+\partial_{\alpha}\left(g^{\sigma \bar{\gamma}} \partial_{\bar{\gamma}} g_{\sigma \bar{\beta}}\right) \\
i \rho_{\beta \alpha}^{b}=(d \theta)_{\beta \alpha}=\partial_{\beta} \theta_{\alpha}-\partial_{\alpha} \theta_{\beta} .
\end{gathered}
$$


16. Chern Connections with holonomy contained in $S U(n)$. Hermitian manifolds which admit a Chern connection with holonomy contained in $S U(n)$ necessarily admit a holomorphic (n,0)-form. Since the existence of holomorphic (n,0)-forms depends only on the choice of complex structure, this can be used to show whether a complex manifold admits a hermitian structure for which the associated Chern connection has holonomy contained in $S U(n)$. To find whether a certain complex manifold admits $(\mathrm{n}, 0)$-holomorphic forms one can use the Kodaira-type vanishing theorems $[27,13]$ together with the results in [22].

For complex manifolds satisfying the $\partial \bar{\partial}$-lemma, we have the following:

THEOREM 8. Let $(M, J)$ be a 2n-dimensional compact connected complex nonKähler manifold with vanishing first Chern class, $c_{1}(M, J)=0$. Suppose $(M, J)$ satisfies the $\partial \bar{\partial}$-lemma. Then

1. i) There exists a Hermitian structure such that the holonomy of the Chern connection is contained in $S U(n), \rho^{c}=0$;

2. ii) The Hodge number $h^{n, 0}=1$.

Proof. The Ricci form $\rho^{c}$ of the Chern connection of any hermitian structure $(g, J)$ represents the first Chern class of the manifold. Therefore since $c_{1}(M, J)=0$, $\rho^{c}$ is exact. Because $\rho^{c}$ is also a $(1,1)$-form, applying the $\partial \bar{\partial}$-lemma, we find that

$$
\rho^{c}=i \partial \bar{\partial} h
$$

for some real function $h$ on $M$. Next we consider the manifold $M$ with hermitian structure $\left(M, \tilde{g}=e^{h / n} g, J\right)$. Using (15.155), we find that the Chern Ricci form $\tilde{\rho}^{c}$ of the new hermitian structure vanishes because

$$
i \tilde{\rho}_{\bar{\beta} \alpha}^{c}=\partial_{\bar{\beta}} \partial_{\alpha} h+i \rho_{\bar{\beta} \alpha}^{c}=0 .
$$

Hence, the holonomy of the Chern connection of $(M, \tilde{g} J)$ is contained in $S U(n)$. This proves (i).

To show (ii), the Gauduchon plurigenera theorem [13] implies $h^{n, 0}(M, J) \leq 1$ since the function $\tilde{u}=\operatorname{trace}\left(\tilde{\rho}^{c}\right)=0$. Since the holonomy of the Chern connection of $(M, \tilde{g}, J)$ is contained in $S U(n)$, there is a parallel (n,0)-form. A parallel (n,0)-form with respect to the Chern connection is necessarily holomorphic. Hence, $h^{n, 0}=1$. This proves (ii).

COROLLARY 6. On $k \geq 2$-copies of $S^{3} \times S^{3}$ there exists a hermitian structure such that the holonomy of the Chern connection is contained in SU(3). In the conformal class of any hermitian structure there exists a unique (up to homothety) one with $\operatorname{Hol}\left(\nabla^{c}\right) \subseteq S U(3)$.

17. Bismut Connections with holonomy contained in $S U(n)$. Consider the following lemma

LEMMA 1. The Bismut Ricci forms $\rho^{b}, \tilde{\rho}^{b}$ of two conformally equivalent hermitian structures $(M, g, J)$ and $\left(M, \tilde{g}=e^{f} g, J\right)$ are related by

$$
i \tilde{\rho}_{\bar{\beta} \alpha}=i \rho_{\bar{\beta} \alpha}+(2-n) \partial_{\bar{\beta}} \partial_{\alpha} f ; \quad \tilde{\rho}_{\beta \alpha}=\rho_{\beta \alpha} .
$$

Proof.. It follows by straightforward calculations from (15.156) and (15.157).

TheOREM 9. Let $(M, J)$ be a 2n-dimensional compact complex non-Kähler manifold with vanishing first Chern class, $c_{1}(M, J)=0$. Suppose $(M, J)$ satisfies the 
$\partial \bar{\partial}$-lemma and that there exists a hermitian structure $(g, J)$ such that $d \theta$ is a $(1,1)$ form. Then there exists another unique (up to homothety) conformal hermitian structure $\left(M, \bar{g}=e^{f} g, J\right)$ such that the holonomy of the associated Bismut connection is contained in $S U(n), \rho^{b}=0$, provided $n \geq 3$.

Proof. Let $(M, g, J)$ be the hermitian structure with $d \theta$ a $(1,1)$-form. Using (15.157), we find that the Ricci form $\rho^{b}$ of the Bismut connection is a (1,1)-form. Therefore it is an exact $(1,1)$-form since $\rho^{b}$ represents the first Chern class which is zero. Applying the $\partial \bar{\partial}$-lemma we can write $\rho^{b}=i \partial \bar{\partial} f$, for some real function $f$ on $M$. Next using lemma 1 , it is straightforward to observe that the Ricci form $\bar{\rho}^{b}$ of the Bismut connection of the hermitian structure $\left(M, \bar{g}=e^{f / 2-n} g, J\right)$ vanishes. Thus, the holonomy of the Bismut connection of $(M, \bar{g}, J)$ is contained in $\mathrm{SU}(\mathrm{n})$. The uniqueness follows since on a compact hermitian manifold the equation $g^{\bar{\beta} \alpha} \partial_{\bar{\beta}} \partial_{\alpha} f=0$ has only constant solutions. This completes the proof.

We remark that if the Lee form $\theta$ is an exact form i.e. the structure is conformally balanced, then the above theorem applies. So we have the following corollary:

Corollary 7. Let $(M, g, J)$ be a 2n-dimensional compact complex balanced nonKähler manifold with vanishing first Chern class, $c_{1}(M, J)=0$. Suppose $(M, J)$ satisfies the $\partial \bar{\partial}$-lemma. Then there exists another conformal hermitian structure $\left(M, \bar{g}=e^{f} g, J\right)$, unique up to homothety, such that the holonomy of the associated Bismut connection is contained in $S U(n)$ provided $n \geq 3$.

A Moishezon manifold is a compact complex manifold which is bimeromorphic to a projective variety. Any Moishezon manifold satisfies the $\partial \bar{\partial}$-lemma by a result of Deligne. Alessandrini and Bassanelli proved in [2], Corollary 4.6 that every Moishezon manifold is balanced. Therefore from the above Corollary 7 we have the following:

Corollary 8. Every Moishezon manifold of complex dimension $n, n \geq 3$, with vanishing first Chern class admits a hermitian structure for which the Chern and Bismut connections have holonomy contained in $S U(n)$. The torsion of such a Bismut connection is not closed.

Proof. We need only to show that a Moishezon manifold cannot admit a strong hermitian structure for which the Bismut connection has holonomy contained in $S U(n)$. For this, we use the result of [22], Corollary 4.2, which asserts the following: if the torsion of a Bismut connection with vanishing Ricci form is closed on a compact non-Kähler hermitian manifold, then there are no holomorphic $(\mathrm{n}, 0)$-forms, i.e. the canonical bundle cannot be holomorphically trivial. But Theorem 8 implies that the Moishezon manifolds with vanishing first Chern class admit a holomorphic $(\mathrm{n}, 0)$-form, a contradiction.

In view of Corollary 8 Moishezon manifolds with vanishing first Chern class may have applications in heterotic strings.

18. Proof of Theorem 1. Let $M$ denote a connected sum of $k \geq 2$-copies of $S^{3} \times S^{3} . M$ is cohomologically Kähler, $b_{1}(M)=h^{1,0}=h^{0,1}=0$ and $h^{3,0}=h^{0,3}=1$ [28]. According to Theorem 4.10 in [22], if there exists a hermitian structure such that the restricted holonomy $\operatorname{Hol}\left(\nabla^{b}\right) \subset S U(3)$, then the structure is conformally balanced i.e. its Gauduchon metric is balanced. Conversely, the existence of a balanced Hermitian structure on $M$ leads to the existence of hermitian structure in the same conformal class with $\mathrm{Hol}\left(\nabla^{b}\right) \subset S U(3)$ by Theorem 9 .

The existence of a balanced hermitian structure on a 2n-dimensional compact complex manifold $(M, J)$ has an intrinsic characterization, namely it can be expressed 
in terms of positive currents by a theorem of Michelsohn [30] which states that a smooth compact complex 2 n-dimensional manifold admits a balanced structure if and only if it is homologically balanced.

We recall that the space of real currents of degree $(n-k, n-k)$ is the dual space of $\Lambda^{k, k}(M)_{R}$, i.e. real (n-k,n-k)-forms with distribution coefficients. A compact complex manifold is balanced if and only if there is no positive current $T$ of degree $(1,1)$ which is the component of a boundary (i.e. if $T=\bar{\partial} S+\partial \bar{S}$ and $T>0$ then $T=0$ [30]). This result has an expression in terms of Aeppli group $V^{1,1}(M)_{R}$. The real $(1,1)$-Aeppli group is defined as

$$
V^{1,1}(M)_{R}=\frac{K e r\left(i \partial \bar{\partial}: \Lambda^{1,1}(M)_{R} \rightarrow \Lambda^{2,2}(M)_{R}\right)}{\left(\partial \Lambda^{0,1}(M)+\bar{\partial} \Lambda^{1,0}(M)\right)_{R}}
$$

The Michelsohn theorem can be rewritten [2]: $M$ is balanced if and only if every non-zero positive $\partial \bar{\partial}$-closed current of degree $(1,1)$ represents a non-zero class in $V^{1,1}(M)_{R}$.

Now, it is clear that any compact cohomologically Kähler complex manifold is balanced. Then, the above mentioned result of Deligne and similar arguments as in the proof of Corollary 8 complete the proof of Theorem 1 .

Corollary 9. Any compact $2 n$-dimensional $(n>2)$ complex manifold with vanishing first Chern class which is cohomologically Kähler admits a hermitian structure with vanishing Ricci form of the Bismut connection. Such hermitian structure is not strong.

19. Examples. Here we shall give examples of Bismut connections with holonomy $S U(n)$ which can be thought of as generalizations of Calabi-Yau manifolds.

Example 1. Consider the $U(n)$ invariant metric

$$
d s^{2}=\left(A\left(r^{2}\right) \delta_{\alpha \bar{\beta}}+B\left(r^{2}\right) \bar{z}_{\alpha} z_{\bar{\beta}}\right) d z^{\alpha} d \bar{z}^{\bar{\beta}}
$$

where $\bar{z}_{\alpha}=\delta_{\alpha \bar{\beta}} \bar{z}^{\bar{\beta}}$ and $z_{\bar{\alpha}}=\delta_{\bar{\alpha} \beta} z^{\beta}$ and $r^{2}=\delta_{\alpha \bar{\beta}} z^{\alpha} z^{\bar{\beta}}$. In this case it can be easily seen that the connection of the canonical bundle is $\omega_{\alpha}=i \bar{z}_{\alpha} f\left(r^{2}\right)$, where

$$
f=(n-1) A^{-1}\left(2 B-A^{\prime}\right)+\left(\log \left(A+r^{2} B\right)\right)^{\prime}
$$

where prime denotes differentiation with respect to $r^{2}$. The condition that $d \omega=0$, necessary for the holonomy to be contained in $S U(n)$, implies that

$$
f=0 \text {. }
$$

We remark that the Kähler case corresponds to taking $B=A^{\prime}$. In this case the solutions produce the Calabi-Yau metrics due to Calabi.

EXAMPLE 2. A compact example of a hermitian manifold for which its Bismut connection has holonomy $\mathrm{SU}(3)$ is as follows. Consider the complex Heisenberg group

$$
G=\left\{\left(\begin{array}{lll}
1 & z_{1} & z_{3} \\
0 & 1 & z_{2} \\
0 & 0 & 1
\end{array}\right) \quad z_{1}, z_{2}, z_{3} \in \mathbf{C}\right\}
$$

with multiplication. The complex Iwasawa manifold is the compact quotient space $M=G / \Gamma$ formed from the right cosets of the discrete group $\Gamma$ given by the matrices 
whose entries $z_{1}, z_{2}, z_{3}$ are Gaussian integers. The 1-forms $d z_{1}, d z_{2}, d z_{3}-z_{1} d z_{2}$ are left invariant by $G$ and by $\Gamma$. These 1 -forms pass to the quotient $M$. We denote by $\alpha_{1}, \alpha_{2}, \alpha_{3}$ the corresponding 1 -forms on $M$, respectively. Consider the Hermitian manifold $(M, g, J)$, where $J$ is the natural complex structure on $M$ arising from the complex coordinates $z_{1}, z_{2}, z_{3}$ on $G$ and the metric $g$ is determined by $g=\sum_{i=1}^{3} \alpha_{i} \otimes \bar{\alpha}_{i}$. The Chern connection $D$ is determined by the conditions that the 1 -forms $\alpha_{1}, \alpha_{2}, \alpha_{3}$ are parallel. The torsion tensor of $D$ is given by $C\left(\alpha_{i}^{\#}, \alpha_{j}^{\#}\right)=-\left[\alpha_{i}^{\#}, \alpha_{j}^{\#}\right], \quad i, j=$ $1,2,3$, where $\alpha_{i}^{\#}$ is the vector field corresponding to $\alpha_{i}$ via $g$. The only nonzero term is $C\left(\alpha_{1}^{\#}, \alpha_{2}^{\#}\right)=-\alpha_{3}^{\#}$ and its complex conjugate. Thus, the space $(M, g, J)$ is a compact balanced Hermitian (non Kähler) manifold with a flat Chern connection and automatically the holonomy group of its Bismut connection is contained in $\mathrm{SU}(3)$ by formula (15.150). The (0,3)-form $\psi=\alpha_{1} \wedge \alpha_{2} \wedge \alpha_{3}$ is parallel with respect to both Chern and Bismut connections. Let $e_{1}, e_{2}, e_{3}, J e_{1}, J e_{2}, J e_{3}$ be a real basis determined by $\alpha_{j}^{\#}=e_{j}-\sqrt{-1} J e_{j}$. Then the real Iwasawa manifold $X$ determined by $e_{1}, e_{2}, e_{3}$ is a SAS calibration with respect to $R e \psi$. It admits moduli since $V=-J e_{3}$ is a SAS deformation. Indeed, $U=J V=e_{3}$ is a Killing vector field on $M$ and therefore it is also holomorphic by results in [11] since the Chern connection is flat. Thus, $e_{3}$ is a SAS deformation of $X$ in $M$.

Note added to the proof. A counterexample to conjecture one has been found in [36] for the case of holomorphically trivial canonical bundle.

\section{REFERENCES}

[1] L. Alessandrini And G. Bassanelli, Positive $\partial \bar{\partial}$-closed currents and non-Kähler geometry, J. Geom. Analysis, 2(1992), pp. 291-316.

[2] L. Alessandrini And G. Bassanelli, Metric properties of manifolds bimeromorphic to compact Kähler manifolds, J. Diff. Geometry, 37(1993), pp. 95-121.

[3] B. Alexandrov and S. Ivanov, Vanishing theorems on Hermitian manifolds, Differential Geometry and its Applications, 14:3(2001), pp. 251-265; math.DG/9901090.

[4] F. Belgun, A. MoroiAnu, Nearly Kähler 6-manifolds with reduced holonomy, Ann. Global Anal. Geom., 19(2001), pp. 307.

[5] J.-M. Bismut, A local index theorem for non-Kähler manifolds, Math. Ann., 284(1989), pp. 681-699.

[6] F.M. Cabrera, M.D. Monar and A.F. Swann, Classification of $G_{2}$ structures, J. London Math. Soc., 53(1996), pp. 407-416.

[7] M. Cvetic, G.W. Gibbons, H. Lu, C.N. Pope, Cohomogeneity one manifolds of Spin(7) and $G_{2}$ holonomy, hep-th/0108245.

[8] N.EJIRI, Totally real submanifolds in a 6-sphere, Proc. Amer. Math. Soc., 83(1981), pp. 759763.

[9] M. Fernandez, A. Gray, Riemannian manifolds with structure group $G_{2}$, Ann. Mat. Pura Appl., 132(1982), pp. 19-45.

[10] Th. FRIEDRICH, S.IVANOv, Parallel spinors and connections with skew symmetric torsion in string theory, Asian J. Math., 6(2002), pp. 303-336; math.DG/0102142.

[11] G. GANCHEV AND S. IVANOV, Holomorphic and Killing vector fields on compact balanced Hermitian manifolds, International Journal of Mathematics, 11(2000), pp. 15-28.

[12] G. GANChEv AND S. IVANOV, Harmonic and holomorphic 1-forms on compact balanced Hermitian manifold, Differential Geometry and its Applications, 14:1(2001), pp. 79-93.

[13] P. Gauduchon, Fibrés hermitiennes à endomorphisme de Ricci non-négatif., Bull. Soc. Math. France, 105(1977), pp. 113-140.

[14] A. GraY, The structure of nearly Kähler manifolds, Math. Ann., 223(1976), pp. 233-248.

[15] A. Gray, L. Hervella, The sixteen classes of AlmostHermitian manifolds and their linear invariants, Ann. di Mat. pura ed appl., 123(1980), pp. 35-58.

[16] R. GRunewald, Six-dimensional Riemannian manifolds with real Killing spinor, Ann. Glob. Anal. Geom., 8(1990), pp. 43-59. 
[17] J.Gutowski, G.PApadopoulos, AdS Calibrations, Phys. Lett., B462:81-88 (1999); hepth/9902034.

[18] J. Gutowski, G. Papadopoulos and P.K. Townsend, Supersymmetry and Generalized calibrations, Phys. Rev., D60:106006 (1999); hep-th/9905156.

[19] R.HaRvey, H.B.Lawson, Calibrated Geometries, Acta. Math., 148(1982), pp. 47.

[20] N.Hitchin, The geometry of three-forms in six and seven dimensions, to appear in J. Diff. Geom., math.DG/0010054.

[21] S.Ivanov, G. PAPAdopoulos, A no-go theorem for string warped compactification, Phys. Lett., B 497 (2001), pp. 309-316.

[22] S. Ivanov, G. PAPAdopoulos, Vanishing Theorems and String Backgrounds, Class. Quantum Grav., 18(2001), pp. 1089-1110.

[23] S. IVANov, Connections with torsion, parallel spinors and geometry of Spin(7) manifolds, math.DG/0111216.

[24] D. JOYCE, Lectures on special Lagrangian geometry, math.DG/0111111.

[25] D. JoyCE, Compact Manifolds with Special Holonomy, Oxford Mathematical Monographs Series, OUP, (2000).

[26] V. KiRichenKo, K-spaces of maximal rank, (russian), Mat. Zam., 22(1977), pp. 465-476.

[27] S. Kobayashi AND H. Wu, On holomorphic sections of certain Hermitian vector bundles, Math. Ann., 189(1970), pp. 1-4.

[28] P. LU, G.TIAN, The complex structures on connected sums of $S^{3} \times S^{3}$, in "Manifolds and geometry (Pisa 1993)", pp. 284-293, Sympos. Math. XXXIV, Cambridge Univ. Press, Cambridge, (1996).

[29] R C McLean, Deformations of calibrated submanifolds, Comm. Anal. Geom., 6(1998), pp. 705-747.

[30] M.-L. Michelsohn, On the existence of special metrics in complex geometry, Acta Math., 143(1983), pp. 261-295.

[31] S. SAluR, Deformations of Special Lagrangian Submanifolds, Commun. Contemp. Math., 2(2000), pp. 365; math.DG/9906048.

[32] I.R ShafareVich, Basic Algebraic Geometry 2, Chapter VIII, Springer-Verlag (1996).

[33] A. Strominger, Superstrings with torsion, Nucl. Physics, B 274 (1986), pp. 254-284.

[34] K. YANO, Integral formulas in Riemannian geometry, Marcel Dekker, INC., New-York 1970.

[35] S.-T.YAU, On the Ricci curvature of a compact Kähler manifold and the complex MongeAmpere equation I, Com. Pure Appl. Math., 31(1978), pp. 339-411.

[36] A. Fino AND G. GRantcharov, On the properties of the manifolds with skew-symmetric torsion and holonomy $S U(n)$ and $S p(n)$, arXiv:math.DG/0302358. 
